\title{
Article
}

\section{Computational Fluid Dynamic Studies of Vortex Amplifier Design for the Nuclear Industry-I. Steady-State Conditions}

Parker, D., Birch, Martin John and Francis, J.

Available at https://clok.uclan.ac.uk/7488/

Parker, D., Birch, Martin John and Francis, J. (2011) Computational Fluid Dynamic Studies of Vortex Amplifier Design for the Nuclear Industry-l. Steady-State Conditions. Journal of Fluids Engineering, 133 (4). 041103. ISSN 00982202

It is advisable to refer to the publisher's version if you intend to cite from the work.

http://dx.doi.org/10.1115/1.4003775

For more information about UCLan's research in this area go to http://www.uclan.ac.uk/researchgroups/ and search for <name of research Group>.

For information about Research generally at UCLan please go to http://www.uclan.ac.uk/research/

All outputs in CLoK are protected by Intellectual Property Rights law, including Copyright law. Copyright, IPR and Moral Rights for the works on this site are retained by the individual authors and/or other copyright owners. Terms and conditions for use of this material are defined in the policies page. 
140 Aldersgate Street,

London, UK

e-mail: darren.parker@telerealtrillium.com

\author{
M. J. Birch ${ }^{1}$ \\ e-mail: mjbirch@uclan.ac.uk \\ J. Francis \\ e-mail: jfrancis1@uclan.ac.uk
}

John Tyndall Institute for Nuclear Research,

School of Computing,

Engineering and Physical Sciences,

University of Central Lancashire,

Preston, UK

\section{Parker \\ John Tyndall Institute for Nuclear Research, \\ School of Computing, \\ Engineering and Physical Sciences, \\ University of Central Lancashire, \\ Preston, UK: \\ Trillium, \\ Computational Fluid Dynamic Studies of Vortex Amplifier Design for the Nuclear Industry -l. Steady-State Conditions}

In this study the effects of changes to the geometry of a vortex amplifier are investigated using computational fluid dynamics (CFD) techniques, in the context of glovebox operations for the nuclear industry. These investigations were required because of anomalous behavior identified when, for operational reasons, a long-established vortex amplifier design was reduced in scale. The aims were (i) to simulate both the anomalous back-flow into the glovebox through the vortex amplifier supply ports, and the precessing vortex core in the amplifier outlet, then (ii) to determine which of the various simulated geometries would best alleviate the supply port back-flow anomaly. Various changes to the geometry of the vortex amplifier were proposed; smoke and air tests were then used to identify a subset of these geometries for subsequent simulation using CFD techniques. Having verified the mesh resolution was sufficient to reproduce the required effects, the code was then validated by comparing the results of the steady-state simulations with the experimental data. The problem is challenging in terms of the range of geometrical and dynamic scales encountered, with consequent impact on mesh quality and turbulence modeling. The anomalous nonaxisymmetric reverse flow in the supply ports of the vortex amplifier has been captured and the mixing in both the chamber and the precessing vortex core has also been successfully reproduced. Finally, by simulating changes to the supply ports that could not be reproduced experimentally at an equivalent cost, the geometry most likely to alleviate the back-flow anomaly has been identified. [DOI: 10.1115/1.4003775]

\section{Introduction}

The basic function of a glovebox in nuclear processes is to protect workers from contact with, or ingestion of, radioactive and hazardous airborne contaminants. The internal environment of such gloveboxes is often air, drawn ultimately from the room in which the glovebox is situated. However, for some critical processes where either fire or moisture is a particular hazard, the gloveboxes are inerted with argon or nitrogen to maintain low concentrations of oxygen and vapor inside the glovebox.

Protection is provided by the mainly transparent glovebox fabric and stainless steel frame, and by the glovebox ventilation system. Together, the fabric and frame form a barrier, but for effective protection it is necessary to maintain a negative differential pressure between the glovebox internal environment and the surrounding area. Typically, gloveboxes are kept at between 250 $\mathrm{Pa}$ and $500 \mathrm{~Pa}$ vacuum pressure. For safety reasons, airflow must be maintained into the glovebox under all operating conditions. The airflow removes moisture, heat, and explosive or corrosive gases from the glovebox. During normal operation the air or inert gas supply flow rate is minimal, but when a breach occurs the airflow rate is immediately increased to ensure a containment velocity of at least $1 \mathrm{~m} / \mathrm{s}$ through the breach. This velocity is sufficient to mitigate the potential for diffusion or convection movement of airborne contaminants against the mainstream flow. The required increase in flow rate can be brought about by means of a vortex amplifier at the glovebox exit.

Gaps in the barrier fabric are intended to be small and, in normal circumstances, the low flow rate into the glovebox is dominated by a controlled in-bleed. The in-bleed valve restricts flow into the

\footnotetext{
${ }^{1}$ Corresponding author.

Contributed by the Fluids Engineering Division of ASME for publication in the Journal OF Fluids EngineERING. Manuscript received July 5 2010; final manuscript received February 3, 2011; published online May 16 2011. Assoc. Editor: Frank C. Visser.
}

glovebox, thereby creating a large pressure drop between the room and glovebox inner space. This describes the normal operating condition in which flow through the glovebox remains laminar, reducing the opportunity for turbulent mixing and the consequent adverse movement of heavier dust. At the glovebox exit, the downstream fan draws fluid into the chamber of the vortex amplifier (VXA) from two sources: (1) from the glovebox, through the VXA radial inlet ports; and (2) from the room, through a filter, an inlet valve, and the VXA tangential control ports. Under normal operating conditions, flow from the two inlets is balanced so that a vortex is established in the VXA chamber, thereby also creating a large pressure drop at the exit from the glovebox.

Always present is the possibility of a sudden breach when a glove is damaged, resulting in loss of depression. The flow path through the breach and glovebox to the VXA chamber then becomes the path of least resistance; flow through the tangential control port is no longer of sufficient momentum to divert the breach flow entering the chamber via the glovebox. Hence, the vortex is lost, or at least substantially reduced in swirl number. The effect is to reduce system resistance, allowing a high flow rate to be drawn through the breach by the fan.

In the UK, vortex amplifiers (VXAs) with a switching characteristic are used to maintain containment in the event of a sudden breach $[1,2]$. Until 2000, the same model of VXA had been used in the nuclear industry for over 20 years. Much of the plant still operating at the Sellafield site continues to use this device, but a model known as the Mini-VXA is now also in service. The Mini$V X A$ was produced by scaling geometrically the previous model, but with the addition of some minor modifications. Subsequent performance of the Mini-VXA has been disappointing, with high oxygen levels noted in the inerted gloveboxes, leading to an expensive increase in the inert gas supply rate [2].

Smoke visualization of airflow on a transparent model of the Mini-VXA indicates a possible mechanism by which air may enter the inerted glovebox via the control and supply ports. However, to 
solve the back-flow problem using smoke visualization and air experiments would be costly and time-consuming. Many tests would have to be performed, each requiring the construction of a different supply port geometry. More costly approaches such as laser Doppler techniques for flow measurement have so far not been used. Issues of health and safety, and laboratory running costs, can be prohibitive. Instead, an alternative approach using computational fluid dynamics (CFD) was adopted to simulate the air-flow through the VXA. The CFD was validated using global pressure and flow data obtained during VXA characterization tests. Using this technique it is possible to investigate the effectiveness on the back-flow problem of modifications to the supply port geometry, without the need to operate expensive experimental test rigs.

The main objectives of the steady state CFD investigation were (1) to reproduce the oxygen leakage shown in the smoke visualization experiment using CFX, and (2) to model the subset of five prototype geometries and thus determine the best solution to the back-flow problem.

\section{The Computational Problem}

It is only in the last 10 or 15 years, with the advent of sufficient computing power at reasonable cost, that the use of CFD has been feasible for engineering studies such as that described herein. High-performance parallel computers are now affordably within the budgets of many research groups, including the John Tyndall Institute for Nuclear Research at the University of Central Lancashire (UCLan). The basic principles of CFD are generic and are applicable to many varied fluid-flow applications, not only device engineering but also other fields of study such as the design of efficient vehicles and engines, the energy balance of buildings, and the evolution of large-scale structures in cosmology. As a result, focus has shifted somewhat from a need for validation against experimental results to confidence in using the tools in a predictive manner, within certain guidelines, provided that the mesh can be verified.

Previous CFD studies of vortex amplifiers are few. One of the earliest numerical models using finite techniques was that of Shimizu et al. [3]. Although no mention is made of CFD, finite differencing techniques were used to solve the full Navier-Stokes equations with primitive variables. Flows were considered to be axisymmetric, incompressible, and laminar. Flow through the majority of VXAs tends to be turbulent for at least some of the characteristic. Isobaric and velocity distributions were presented for horizontal sections through the vortex chamber and for vertical sections through chamber and diffuser. Results clearly show a parabolic area forming in the vortex chamber at high swirl conditions, where axial flow is effectively reduced to zero. This confirms the results of the visual investigation conducted by Wormley [4], where a doughnut-shaped flow formation was observed in the vortex chamber (also referred to as a "spinning doughnut").

Boucher et al. [5] used a commercial CFD package to solve the time-averaged Navier-Stokes equations, modeling flow through a short vortex chamber. Turbulence was simulated using the twoequation $\kappa-\varepsilon$ model to calculate kinetic energy and dispersion. The purpose of the simulation was to investigate flow in the VXA chamber at high and low swirl conditions. The chamber was modeled about the mid-plane in two dimensions, with geometric symmetry being used to reduce the physical scale of the model. Isobaric lines and velocity vectors were plotted for both conditions. It was noted that under high swirl conditions the parabolic area identified by Wormley [4] was not predicted by the model. However, agreement with laser Doppler anemometry readings was found to be good, although tangential velocity discrepancies for both high and low swirl conditions were in the region of $19 \%$. One potential explanation for the missing forced vortex area in the CFD simulation was the absence of a front and back chamber wall. The simulation was based upon a two-dimensional grid situ- ated in the mid-plane of the chamber. The only boundary walls present were those created by the circumference of the chamber and the control/supply ports. Without the front and rear chamber walls there would be no radial flow through the boundary layers created by viscous action with the walls. The work of Boucher et al. [5] may be considered important since it was successful in predicting two-dimensional flows, and because the missing "doughnut" implies strongly that in practical (and thin-chambered) devices the problem is one of three dimensions.

A more ambitious time-dependent CFD modeling exercise was carried out by Woolhouse et al. [6]. The focus of the study was to simulate the flow structures in the chamber of a turn-up vortex amplifier (TuVA). Commercial CFD software was used to model flow at high and low swirl conditions. Both $k-\varepsilon$ and Reynolds Stress Models (RSM) were used to simulate turbulence. Both models proved to be less accurate at zero swirl values. However, the percentage discrepancy for the RSM model under full swirl conditions was between $1.3 \%$ and $1.8 \%$. Poor agreement with experimental values was thought to be the result of manufacturing errors in the fabrication of the TuVA's supply and control ports, the theoretical model performing better than the actual TuVA. Time-dependent solutions were sought to try and account for the dynamic effects of the complex flows within the exit port. Velocity vector plots for the device show an off-center vortex core (possibly a precessing vortex core). Results from the simulations were encouraging, but time to convergence for the RSM model varied from 10 days to 5 weeks.

More recent studies of Ranque-Hilsch vortex tubes have used CFD to produce two-dimensional axisymmetric simulations of the flow fields within the tubes. Aljuwayhel et al. [7] used CFD to investigate flow phenomena in a counter-flow vortex tube. The device itself was similar to a vortex diode, in that a tangential inlet was used to establish a forced vortex or quasi-solid rotation; as flow was drawn down the tube toward the axial outlet a free vortex or potential vortex flow field was established. This two-dimensional steady state simulation was developed using an industry standard CFD software package (FLUENT). The simulation consisted of 45,000 grid elements. Various turbulence models were used, including a $\kappa-\varepsilon$ model with a turbulent Prandtl number equal to unity and a renormalization group theory (RNG) $\kappa-\varepsilon$ model that calculated a custom Prandtl number for the flow condition. Comparisons with experimental data were good, with an overall energy balance of better than $7 \%$.

Later, Upendra-Behera et al. [8] used CFD analysis to establish an optimum vortex tube geometry based upon a variation in tangential nozzle profiles, tube length, and diameter. The STAR-CD CFD package was used to produce a three-dimensional simulation of the vortex tube made up of approximately 750,000 elements. Although the mesh used was of good quality, planes of symmetry were used to reduce the overall size of the model and, hence, decrease the time taken to obtain a solution. This is a common approach when modeling rotating flows (however, it is questionable in this case, as vortex flows have been shown to precess around the periphery of enclosures). The transient nature of the vortex flow field and its motion around the pipe would negate the use of a plane of symmetry. While investigating the effects of geometry on performance of the tube, Upendra-Behera et al. also verified the existence of a secondary circulation flow superimposed upon the primary forced vortex [8]. Similar recirculation flows exist within the VXA at high values of swirl. However, invasive measurement techniques to date have proved to be ineffective due to disruption of the flow field.

Wang and Priestman [9] simulated the behavior of a symmetrical turn-up vortex amplifier (an eight-port STuVA) to obtain insight into its maximum throughput and to understand the relation between its design parameters and flow characteristics using different turbulent models. They found that the RSM gave predictions slightly closer to the experimental data than the other models, although the renormalized $\kappa-\varepsilon$ model predicted nearly as accurately as the RSM. They both improved errors by about $3 \%$ 
compared to the standard $\kappa-\varepsilon$ model but took a long time for convergence. In their study it was determined that a good combination was the RSM, together with the two-layer wall model and a higher order discretization scheme, which improved accuracy by more than $10 \%$ compared to the standard $\kappa-\varepsilon$ model.

It is the success of these previous simulations that has led to the adoption of CFD modeling to study the back-flow problems identified with the Mini-VXA. The intention is to use CFD in a predictive manner to establish which prototype geometry is most likely to reduce the back-flow. The challenge is substantial:

- Without laser or hot wire measurements at the VXA ports, coupled with the presence of curvature and swirl in the VXA chamber, it is necessary to establish the upstream boundary in the glovebox, and the downstream boundary some distance down the exit piping. This creates large-scale differences within the problem that result in:

- a need to carefully select wall functions, and monitor transition and growth quality criteria using an unstructured mesh

- very slow moving fluid in the glovebox where residuals were difficult to reduce

- a wide range of Reynolds number being experienced at various locations within the flow domain, with both laminar and turbulent sections of flow (the dynamics and geometry in different regions lend themselves to different turbulence models)

- The generic vortex amplifier device is one of a class of devices known to generate a precessing vortex in the exit port, introducing some periodicity into a relatively steady flow. Other sources of instability in the device include a complex swirling three-dimensional flow in the chamber, where a spinning doughnut has been observed, this being a region of flow where the residence time of a particle can be considerably longer than the average residence time calculated from global flow measurements. The swirl numbers for the current device are marginally smaller than those for which a full spinning doughnut has been observed by others, and some complexity of flow is therefore expected.

Nonetheless, steady state results have been useful in establishing the most promising prototype and have led to further experimental studies and field trials of a solution that significantly reduced the back-flow problem. However, this paper focuses on the computational study. One unexpected result was that each supply port has a unique fluid flow structure (i.e., the fluid flow in the plane of the VXA chamber is orthogonally asymmetric). This has led to a hypothesis that the asymmetric supply port flow structures may be the result of the precessing vortex core (rather than a numerical or mesh-generated effect), and that an additional transient study was therefore needed to capture the precession and confirm any link between this and the supply port flow structures.

The development of the CFD models and simulations was carried out in accordance with the best-practice guidance published by ANsys [10], MARNET CFD [11], and The American Institute of Aeronautics and Astronautics (AIAA) [12]. The CFD terminology used throughout this paper has been based upon the definitions published by the AIAA.

It should be noted that only steady-state simulations are considered herein, and that time-dependent transient studies of the vortex amplifier will be reported in a subsequent accompanying paper.

\section{The Operating Principle of the VXA}

Ventilation systems for nuclear plants must have very high reliability and effectiveness. In this application fluidic systems have advantages that traditional systems lack. Fluidic systems will not easily wear out, have a relatively fast response, and in some cases may be cheaper than an equivalent conventional system. Most importantly they have fewer moving parts, usually none, so they are inherently more reliable provided that the fluidic design is effective.

The principle of the VXA combines the concept of a reverse flow vortex diode with that of a diverter valve, consisting of a thin cylindrical chamber with supply and control inlet ports, and one outlet port. The basic principle of operation is illustrated in Fig. 1 in which there are three orthogonal ports: supply (connected to the glovebox), outlet (connected to the vacuum pump), and control (open to atmosphere, via a filter). The right-hand panel shows normal operation with balanced supply and control flows causing a vortex within the chamber; this results in a higher resistance to outlet flow. The left-hand panel shows the case of a major glovebox breach in which the supply flow swamps the control flow, collapsing the vortex and thus greatly reducing the resistance to outlet flow. Practical versions of the VXA are geometrically axisymmetric, with four control and supply ports arranged in quadrature around the axial outlet port.

\section{Experimental Evidence for the Oxygen Back-Flow Problem}

4.1 Visualizing the Back-Flow Problem Using Smoke Tests. For safety reasons, gloveboxes used in the nuclear industry are often purged with an inert gas, usually nitrogen. When the Mini-VXA was first tested in this context, it was found that the glovebox environment was contaminated with oxygen, and that the oxygen concentration increased towards the supply ports. Air carries moisture and supports combustion. The safety implications of an oxidizing environment within the glovebox meant that a solution to this problem had to be found.

Smoke visualization tests on a mock VXA and glovebox have identified a mechanism by which air can enter the glovebox from the control ports via the supply ports, against the overall direction of flow. In these tests the glovebox is not purged with nitrogen; air is used, and orange smoke is drawn into the control ports. The tests began with the valves to the control ports closed and the air supply to the glovebox fully open for maximum purge flow. In this situation, the air flowed through the glovebox and entered the VXA through the four radial supply ports, whereupon it proceeded radially through the VXA directly to the outlet on the back wall at the center of the chamber. This emulates the emergency condition of minimum resistance in which a major breach has occurred, such as when a glove is punctured, but without oxygen ingress.

As the control port valve is opened, air (and therefore smoke) is drawn through the four control ports which are situated at $90^{\circ}$ to the supply ports, tangential to the chamber. The momentum of the air passing through these ports deflects the supply from the glovebox, creating a vortex in the chamber. The vortex strength grows as the control port valve is gradually opened. Fig. 2(a) shows this condition in which both the supply (i.e., glovebox inlet) and control valves are fully open. Smoke enters the VXA by the control ports and the vortex is apparent.
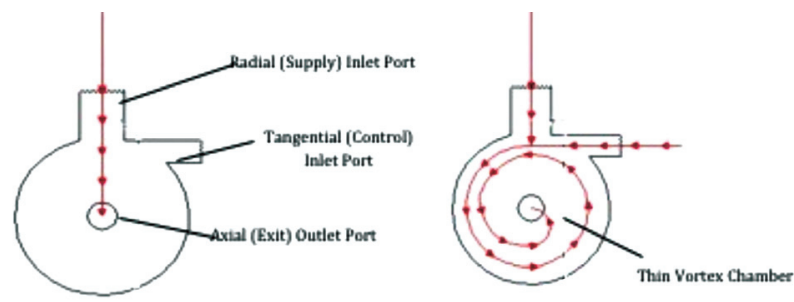

Radial Flow Only

No tangential flow, low resistance to radial flow.
Radial and Tangential Flow Tangential flow applied, vortex established. High resistance to radial flow.

Fig. 1 The principle of operation of the vortex amplifier 

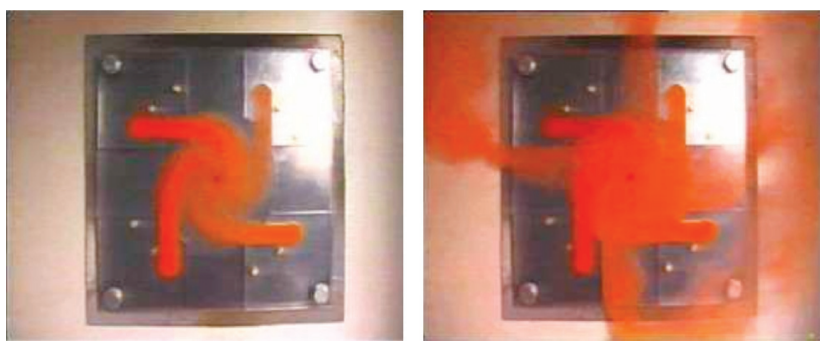

Fig. 2 VXA smoke tests showing supply port back-flow: (a, left) control and supply ports fully open; (b, right) restricted supply reveals back-flow

When the control port valve is fully open, the ratio of supply to control port momentum is further decreased by gradually closing the valve on the air supply to the glovebox. As the supply valves are closed it is observed that the vortex strength increases. In this situation the smoke from the control ports is carried progressively further across the supply ports until a point is reached where it is carried all the way across to each supply port wall/chamber corner. When this happens a sudden transitional change occurs, and a recirculating zone appears in the supply ports, which with further supply restriction quickly grows back down the wall of the supply port into the glovebox. Figure $2(b)$ shows this condition where supply to the glovebox has been restricted. Smoke enters from the control ports but the smoke has moved back up the radial supply ports and, hence, into the glovebox.

This is the most likely mechanism by which air enters an inerted glovebox. Current research is aimed at identifying more clearly the limits of geometry and fluid properties under which this can occur by modeling the VXA and glovebox using CFD. However, before the CFD studies were carried-out, air tests were used to learn more about the problem and to identify a subset of possible geometrical solutions from an initial set of proposals.

4.2 Air Tests for Various Supply and Control Port Geometries Having demonstrated the back-flow effect in the smoke tests, modifications to the geometry of the control and supply ports were proposed to alleviate the problem. Five different control port geometries were defined, each of varying shape and width. In addition, 25 supply port geometries were also created by applying varying degrees of taper, both converging and diverging, to the supply port walls which face each control port. Each supply port geometry was tested against all the control port variations, and vice versa, using air as the fluid medium. It was found that changes to the control ports had little effect and that the original geometry was the best form to adopt for CFD studies. Regarding the supply ports, it was realized that the diverging taper was ineffective and that 5 of the 25 converging tapers (Fig. 3) should subsequently be simulated. The geometries selected (identified as 2.2, 2.3, 3.2, 3.3, and 7.0) are summarized in Table 1 and Table 2.

\section{Modeling the Mini-VXA using CFX}

5.1 Hardware and Software Platform. Two alternative CFX v11.0 platforms were available as the study progressed: initially, a PC cluster was used, comprising 7 Dell Precision 470 Intel Xeon $3 \mathrm{GHz}$ processors; later, 5 stand-alone Antec PCs were also made available, each incorporating dual 64-bit Intel Xenon processors. As well as the single-processor solver installed on each PC, a multiprocessor implementation of v11.0 was also installed on a parallel high-performance SGI Altix 3700 platform running standard Linux. The Altix platform comprised 56 Intel Itanium-2 CPUs (of which 9 CPUs were licensed for use by the authors).

5.2 Geometry Development. The VXA geometries were assembled using ANSYS Design Modeler ${ }^{\mathrm{TM}} 12.0$ which is a solid modeling tool designed to allow the user to build three-dimensional constructs from two-dimensional sketches on predefined planes. The geometry of the VXA was recreated using the original drawings and verified against the actual physical dimensions, which include both the fixed parameters (Table 1) and those which vary (Table 2) for the subset of five supply port geometries selected from the air tests in subsection 4.2. Consequently, the parameters in Table 2 were defined as variables in the CFX Geometric Modeler.

Issues associated with placing domain boundaries close to areas of high swirl, large curvatures, or high pressure gradients are expounded in various best practice guides [10-13]. Given that data pertaining to exact boundary conditions at the VXA's inlets and outlets were not available, the domain boundaries of the model were moved away from these locations to areas where the actual conditions could be specified more accurately. By relocating the domain boundaries, velocity profiles at the VXA's supply

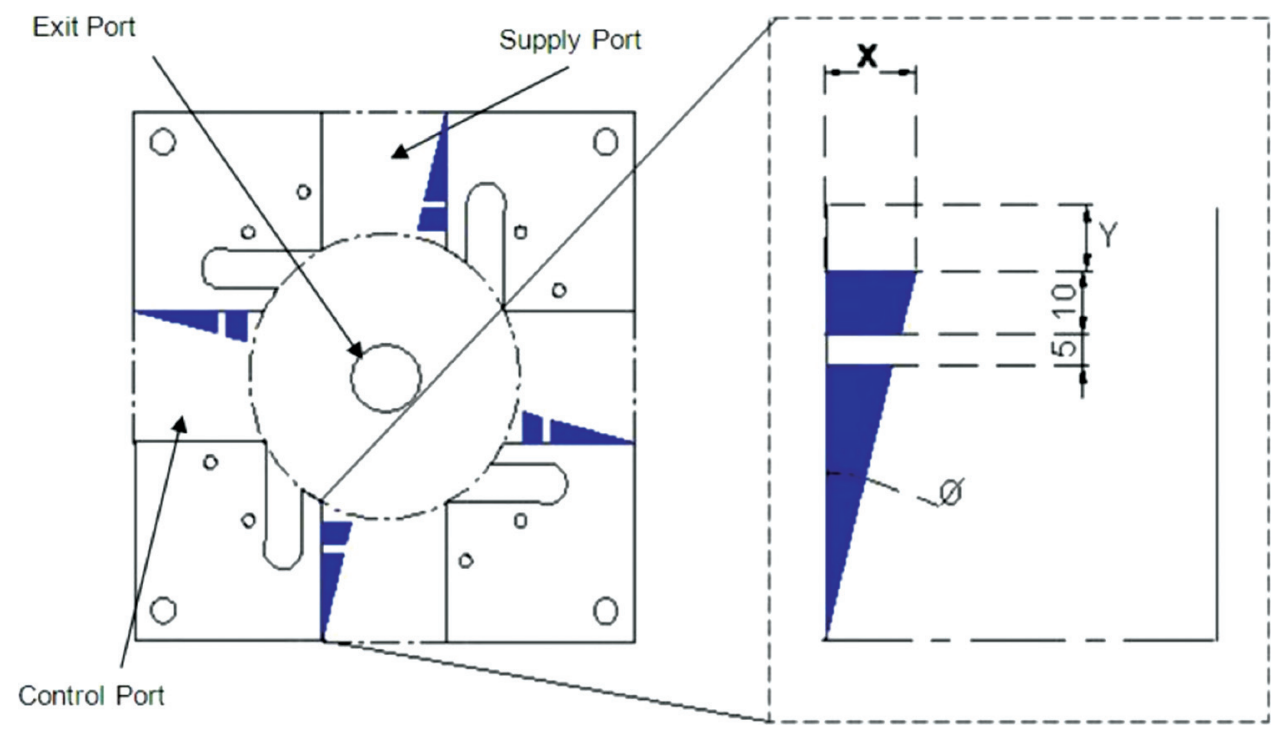

Fig. 3 The vortex amplifier in the plane through the main chamber, showing the geometry of the converging supply port taper and the associated parameters $(X, Y, \Phi)$ in Table 2. (Measurements 5 and 10 are in millimeters; the distribution around the chamber of supply ports SP1-4 is shown in the left-hand panel.) 
Table 1 Fixed VXA parameters

\begin{tabular}{llc}
\hline \hline Parameter & \multicolumn{1}{c}{ Description } & Value \\
\hline$A_{e}\left(\mathrm{~mm}^{2}\right)$ & Area of exit port & 660.6 \\
$A_{c}\left(\mathrm{~mm}^{2}\right)$ & Area of control ports & 760 \\
$r_{0}(\mathrm{~mm})$ & Vortex chamber radius & 69.9 \\
$r_{e}(\mathrm{~mm})$ & Exit port radius & 14.5 \\
$h(\mathrm{~mm})$ & Height of vortex chamber & 11.4 \\
$A_{c} / A_{e}$ & Ratio of control port to exit port areas & 1.151 \\
$r_{0} / r_{e}$ & Ratio of vortex chamber radius to exit port radius & 4.828 \\
$h / r_{e}$ & Ratio of vortex chamber height to exit port radius & 0.786 \\
$h / r_{0}$ & Ratio of height to radius of vortex chamber & 0.163 \\
\hline \hline
\end{tabular}

and exit ports were allowed to develop naturally. In the case of the VXA exit port, the domain boundary was moved some distance down the outlet piping. In the case of the VXA supply ports, the domain boundary was moved back by re-creating part of the glovebox. Figure 4 shows the fluid in the model both with and without the glovebox (only the fluid was modeled because the device has no moving parts).

Though the Mini-VXA is orthogonally symmetrical about the central axis of the outlet port, flow through the device was found to be asymmetric, particularly when operated in its high swirl condition (the area of interest for this study). The precessing vortex core [14] is a periodic feature of the outlet port which introduces asymmetric flow phenomena in the outlet piping that could reduce the accuracy of the solution, preventing or delaying convergence. For this reason the fluid geometry was modeled in its entirety. Though more costly in computational time, it reduced the uncertainty of the model.

5.3 Mesh Development. Each fluid volume was approximated using ANSYS CFX-MESH 11.0. CFX-MESH is a tool for creating unstructured computational meshes which divide each fluid volume into tetrahedra, prisms, and pyramids. The software also includes several facets for checking the quality of both the imported geometry and the mesh. Significant difficulties were encountered by the authors when trying to generate a mesh which satisfied the guidance published by ANSYS. In particular, accurate resolution of the boundary layers in the thin vortex chamber and exit port areas proved to be very time-consuming. Where geometric features varied significantly, local edge length control was used to refine the mesh and achieve the necessary resolution.

Specific, composite two-dimensional surfaces on each VXA geometry were named as regions to enable local mesh controls to be applied. Local mesh settings were applied to the majority of the surfaces on the VXA. The physical parameters of each region and the predicted flow conditions were used to determine the mesh length scale for each region. Further refinement was sometimes required to overcome quality issues with the mesh.

Velocity gradients are significant perpendicular to the surface of the walls. A very fine-volume mesh was generated using inflated boundary layers in order to fully resolve the VXA. The depth of inflation and the number of inflated layers were specified such that the boundary layers on every internal surface within the VXA were bridged by more than 10 nodes. A minimum of 12

Table 2 Geometry-dependent VXA parameters (see Figure 3 for definitions of $X, Y$ and $\Phi$ )

\begin{tabular}{lccccc}
\hline \hline Geometry & $\boldsymbol{A}_{\boldsymbol{s}}(\mathbf{m m} \mathbf{2})$ & $\boldsymbol{X}(\mathbf{m m})$ & $\boldsymbol{Y}(\mathbf{m m})$ & $\Phi(\mathbf{d e g})$ & $\boldsymbol{A}_{\boldsymbol{s}} / \boldsymbol{A}_{\boldsymbol{e}}$ \\
\hline $\mathbf{7 . 0}$ & 2600 & 0 & 0 & 0 & 3.936 \\
$\mathbf{2 . 2}$ & 2200 & 10 & 10 & 10.68 & 3.331 \\
$\mathbf{2 . 3}$ & 2200 & 10 & 20 & 11.77 & 3.331 \\
$\mathbf{3 . 2}$ & 2000 & 15 & 10 & 14.5 & 3.028 \\
$\mathbf{3 . 3}$ & 2000 & 15 & 20 & 17.35 & 3.028 \\
\hline \hline
\end{tabular}

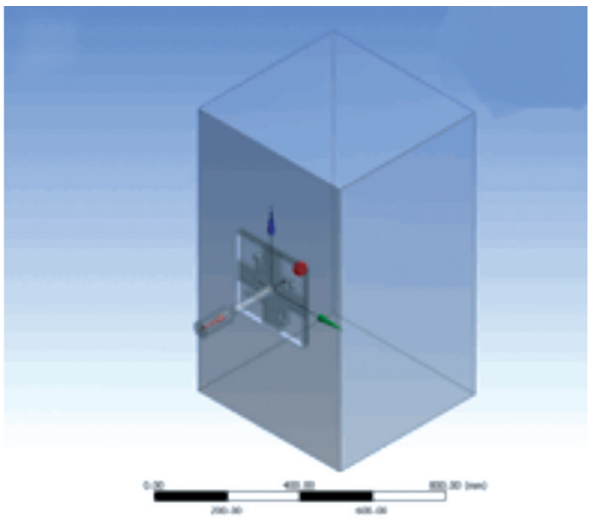

(a)

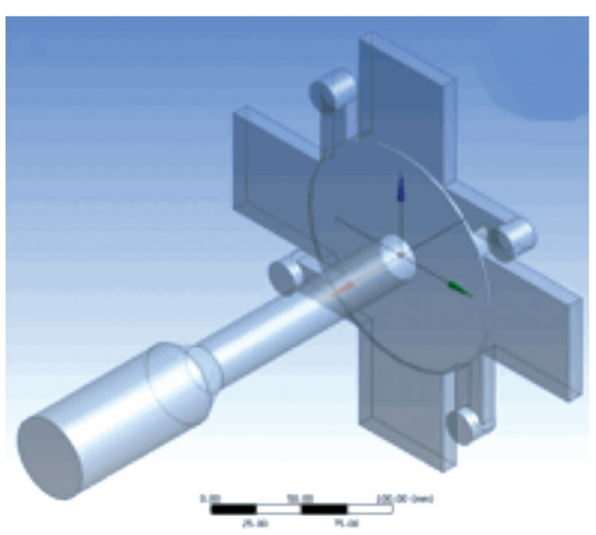

(b)

Fig. 4 Completed fluid geometry for VXA and glovebox

inflated layers were specified for each internal surface, based upon a mesh expansion factor of 1.2. Estimates of boundary thickness were based upon correlations for a flat plate. The one drawback to this method was that the CFX-MESH software could only apply a single common first layer thickness to all faces selected for inflation. This necessitated the application of an over-refined mesh to those areas of the model with larger boundary layers. The vortex chamber required the most refinement to enable the resolution of the boundary layers on the front and back walls. Further refinement of the inflated elements had to be made to create a gap of sufficient size down the center line of the chamber for the volume mesher to function. Some further adjustment of the local surface mesh scale lengths was also required to reduce edge-length and aspect ratios of the inflated elements.

Figure 5 illustrates the results of the automated meshing process. CFX-MESH can only be used to view surface mesh; however, volume mesh can be viewed in the postprocessing package CFXPOST, which also contains the tools for evaluating mesh quality and for visualizing elements that fall outside the recommended quality parameters.

5.4 Definition of the Initial Physical Conditions for the VXA-Glovebox Fluid Environment. The fluid used in the model was air at $25^{\circ} \mathrm{C}$. The initial conditions for the domain and boundary physics are summarized in Table 3 and Table 4, respectively. These tables were produced automatically by the reporting utility within CFX. As can be seen from Table 3, a nonbuoyant isothermal heat transfer model was chosen because the dominant force driving the air in the glovebox and VXA is not convection but pressure. The Baseline (BSL) Reynolds Stress Model with automatic wall treatment was used to approximate the effects of turbulence and boundary layer interaction, and the physical structure of the VXA was assumed to be static (that is, noninertial). Initially, 


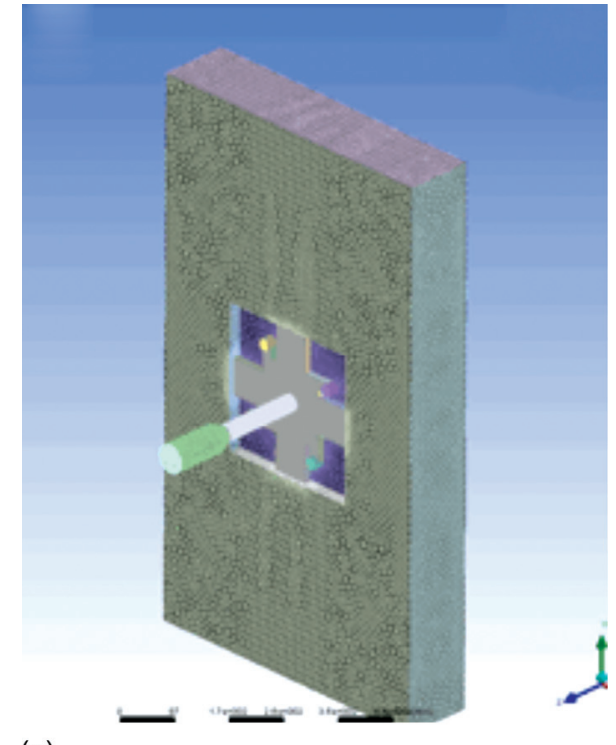

(a)

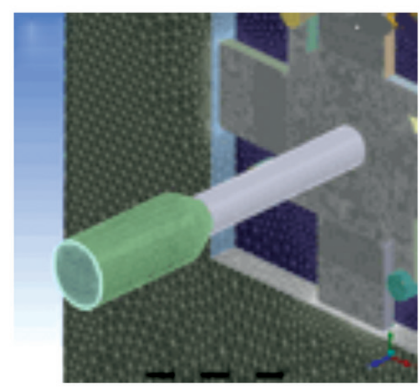

(b)

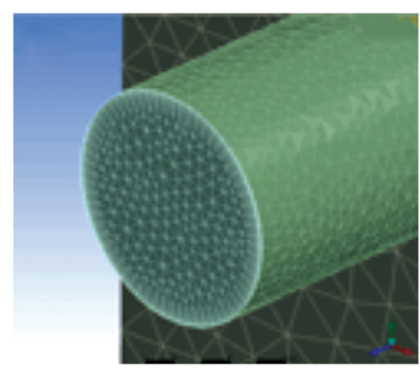

(c)

Fig. 5 Unstructured surface and volume mesh for the fluid space

convergence was deemed to have been achieved when the maximum residual was less than $1 \times 10^{-4}$ and the global imbalances for mass and momentum were $<1 \%$. The time to converge the solutions varied between 49 minutes and 6 hours 30 minutes, depending upon the geometry being simulated. In the majority of instances, convergence occurred within 1500 iterations.

From the relative pressures in Table 4, and allowing for a control port pressure range of -374 to $-370 \mathrm{~Pa}$, the pressure at the outlet was depressed by between 164 and $168 \mathrm{~Pa}$ relative to the

Table 3 Domain physics for VXA-glovebox model

Heat transfer model

Turbulence model

Turbulence wall functions

Buoyancy model

Domain motion
Isothermal

BSL Reynolds Stress Automatic

Nonbuoyant

Stationary
Table 4 Boundary physics for VXA-glovebox model

\begin{tabular}{|c|c|}
\hline \multicolumn{2}{|c|}{ Control ports (opening) } \\
\hline Flow direction & Normal to boundary condition \\
\hline Flow regime & Subsonic \\
\hline Mass and momentum & Opening pressure and direction \\
\hline Relative pressure & -374 to $-370 \mathrm{~Pa}$ \\
\hline Turbulence & Zero gradient \\
\hline \multicolumn{2}{|c|}{ Supply inlet (opening) } \\
\hline Flow regime & Subsonic \\
\hline Mass and momentum & Static pressure for entrainment \\
\hline Relative pressure & $-388 \mathrm{~Pa}$ \\
\hline Turbulence & Zero gradient \\
\hline \multicolumn{2}{|c|}{ Outlet (outlet) } \\
\hline Flow regime & Subsonic \\
\hline Mass and momentum & Average static pressure \\
\hline Relative pressure & $-538 \mathrm{~Pa}$ \\
\hline Pressure averaging & Average over whole object \\
\hline \multicolumn{2}{|c|}{ VA11 default (wall) } \\
\hline Wall influence on flow & Nonslip \\
\hline
\end{tabular}

control ports, and by $150 \mathrm{~Pa}$ relative to the supply ports. Consequently, the flow from the control to the supply ports was driven by a pressure difference of between 14 and $18 \mathrm{~Pa}$.

With the exception of the domain walls, which were specified as nonslip, the remaining boundaries were specified as openings. The entrained flow setting with fully formed turbulence was used to describe the control and exit ports; the glovebox face (incorporating the supply ports) was specified using a total pressure setting with low flow turbulence perpendicular to the boundary. Upon loading the computational mesh, the volume was defined as an isothermal fluid domain (properties of air at $25{ }^{\circ} \mathrm{C}$ and $101.325 \mathrm{kPa}$ absolute pressure). The domain was specified as stationary and buoyancy was neglected. A depression of $-385 \mathrm{~Pa}$ was specified at the glovebox inflow boundary, with normal flow, a turbulent intensity of $1 \%$, and viscosity ratio equal to 1 . A static pressure of $-538 \mathrm{~Pa}$ was specified at the exit boundary. Fully developed turbulence conditions were assumed in the outlet piping, with the boundary condition set to zero gradient. The control ports were specified as openings with a static pressure of $-376 \mathrm{~Pa}$.

It was recognized that the above combination of pressures was not the most robust, the mass flow rate being part of the solution. However, the pressures specified were those previously used during the laboratory testing of the Mini-VXA.

5.5 Turbulence Model and Wall Functions. The additional computational resources required by Reynolds Stress Models (RSMs) to capture the anisotropic turbulent stresses and body forces associated with high swirling flows are well documented (e.g., Refs. $[6,9,15])$. For this reason, two-equation turbulence models such as $\kappa-\varepsilon$ were not used in previous studies because the turbulence equations failed to accurately predict the anisotropy of strain and Reynolds stresses in high swirl flows. More recent advances in computer processing technology mean that the once onerous convergence times associated with RSMs are now less significant. The numerical instabilities associated with the models can be mitigated by using the more robust two-equation models to produce a set of initial values on which the RSM operates. This approach was taken in this study. A baseline RSM was selected for the current study, in which a hybrid $\kappa-\varepsilon$ and $\kappa-\omega$ model was used to produce initial values. In the fully turbulent free-stream areas of flow the hybrid model uses the $\kappa-\varepsilon$ equations. As the flow approaches a wall, blending functions are used to switch to the $\kappa-\omega$ turbulence treatment.

The BSL Reynolds Stress Model is approximately 2.5 times more computationally expensive to run than the two-equation shear stress transport (SST) model. However, its ability to capture anisotropic stress and strain in complex flows such as those that 
exist within the VXA was considered essential to the overall quality of the analysis results.

Automatic wall treatment is available in the ANSYS CFX package for turbulence models incorporating the turbulent frequency equation $(\omega)$. The $\kappa-\omega$ model does not require the same use of complex damping functions to prevent excessive generation of turbulent energy in areas with low Reynolds numbers (for example, near walls). Hence, it can be used to simulate turbulent effects through the boundary layer, all the way down to the wall through the viscous sublayer. This near-wall treatment is considered an advantage in capturing boundary layer effects within the relatively thin VXA chamber. Moreover, the ANSYS literature recommends this wall treatment for provision of the most accurate near-wall results over a wide range of mesh length scales. For this reason, and for the improved boundary layer resolution, the automatic wall treatment methodology was adopted for all simulations.

5.6 Solution Algorithm. Discretization of the domain was carried out using an automated unstructured tetrahedral meshing tool. The solver uses the process of median-dual tessellation to create control-volumes by joining the centroids formed by the midpoints along the edge of each element face. It is these volumes which are then used for the discretization process, the partial differential equations being integrated over each control volume. Using Gauss's divergence theorem, the discretized equations of control volumes adjacent to the boundaries of the domain are adjusted to incorporate the boundary conditions.

When discretizing volumetric terms, approximate values are assigned to each sector surrounding the node before being integrated over the whole control volume. Within the ANSYs solver there are several different schemes to approximate the value of the advection term at the point of integration on the control volume sector, which are:

- first-order upwind differencing scheme;

- numerical advection correction scheme;

- central differencing scheme; and

- high-resolution scheme

On unstructured grids such as those used herein, upwind differencing schemes are known to be susceptible to errors generated from misalignment of the flow vector with the surface of the control element (i.e., the mesh is not orthogonal). The upwind scheme is bounded and conservative. However, it is only accurate to the first order due to the truncation of the Taylor series used to derive the approximation. For this reason it was not selected. The numerical advection scheme is a slightly more sophisticated version of the first-order upwind scheme, in that the model corrects for some of the errors produced by it. While it is accurate to the second order, it is known to introduce dispersive discretization errors into the solution. ANSYS does not recommended this scheme for use with the turbulence models selected by the author. Central differencing schemes, although conservative, are not necessarily bounded; they have the advantage of being accurate to the second order but do not take account of the direction of the flow. The overall stability of the scheme is a combination of the fluid properties, flow properties, and mesh length; hence it is not as reliable as the other schemes. The high-resolution scheme was selected. The scheme is recognized as being conservative and bounded; accuracy is augmented as second-order approximations are used as a default option. The scheme only reverts to first order approximations in areas of discontinuity (unlikely in the subsonic flows here encountered).

The Ansys CFX package uses an iterative strategy to solve the discrete sets of linear algebraic equations. This basically requires the application of a simple algorithm (e.g. the Gauss-Seidel method). Convergence is accelerated through the use of an algebraic multigrid technique. One of the biggest problems with iterative solvers is that convergence times increase as mesh length scales are reduced. ANSYs use an algebraic multigrid technique known as 'Additive Correction'. Upon starting the solver the system of equations for the fine mesh are initially used to reduce the short wavelength error components. The results are then transposed into the course mesh equations, which are then used to reduce the long wave length error components. The final converged solution is then transposed back on to the fine mesh.

5.7 Mesh Verification. An estimate of the discretization error was achieved by carrying out mesh convergence studies on geometries 7.0, 1.1, 1.4, 3.1, and 3.4. A grid refinement approach was used $[10,13,16]$. A coarse mesh solution and three finer meshes, all within the asymptotic range, were generated using the global scaling facility in CFX-MESH. The result was a relatively uniform refinement of the mesh throughout the domain. Where individual mesh length scales had been applied to the surface of specific regions they were manually adjusted to achieve uniformity with the global setting. In accordance with ANSYS recommendations, no alterations were made to the inflated boundary layer settings. The fractional discretization error $\left(A_{\mathrm{rms}}\right)$ was calculated as follows [10]

$$
A_{\mathrm{rms}}=\frac{\left|\Theta_{1}-\Theta_{2}\right|}{\left|\Theta_{1}\right|} \frac{1}{\left(r^{p}-1\right)}
$$

where $\Theta_{l}$ is the value of the initial course grid solution; $\Theta_{2}$ is the value of the refined mesh solution; index $p$ is the formal order of accuracy; and $r$ is the effective mesh refinement factor for an unstructured mesh. The value of $r$ was derived from the variance in grid points between the two meshes and the number of dimensions $(D)$ being modeled in the problem, as follows

$$
r_{\text {effective }}=\left(\frac{N_{1}}{N_{2}}\right)^{1 / D}
$$

For each subsequent mesh refinement the global mesh length scale was reduced from 1.75 in increments of 0.25 . While the linear length scale of the mesh was amended both globally and locally, the overall impact of the changes in relation to the number of elements and nodes was still subject to the uncertainties of the unstructured surface and volume meshing routines. Overall convergence for the steady state simulations was deemed to have been achieved when the maximum residual values for momentum $\left(U_{\text {mom }}, V_{\text {mom }}, W_{\text {mom }}\right)$ and mass $\left(P_{\text {mass }}\right)$ were $1 \times 10^{-4}$, and global balances were less than $1 \%$. In practice it proved to be very difficult to achieve this residual figure.

The results of the mesh verification are shown in Fig. 6, in which it is clear that the fractional discretization error has attained asymptotic behavior within the chosen range of mesh resolution. The data used to calculate the spatial discretization errors were based upon absolute mass flow rates at the exit and control port openings.

On average the variance between $A_{\text {rms }}$ for the two smallest mesh lengths was less than $8 \%$. The solution was considered to be qualitatively mesh independent when the number of elements in the models approached $2 \times 10^{6}$. In the interests of computational efficiency, and with little to be gained from using the grids with the smaller mesh length scales, this mesh resolution was adopted for the remainder of the simulation exercises.

\section{Results of the Steady-State Simulation}

In presenting these results, we first verify that the VXA geometries identified for further study by the air experiments behave in reasonable compliance with their respective simulations (Sec. 6.1). We then use the representative geometry 7.0 to demonstrate the behavior of the simulated VXA in three regions of the device: 


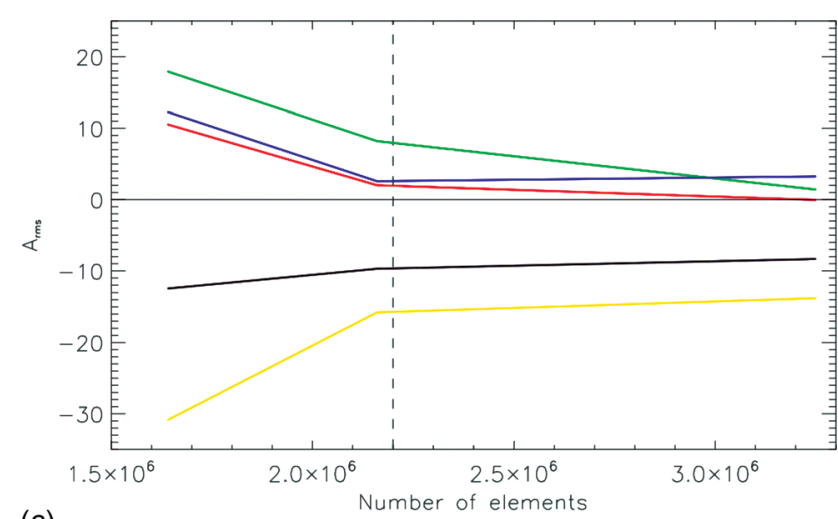

(a)

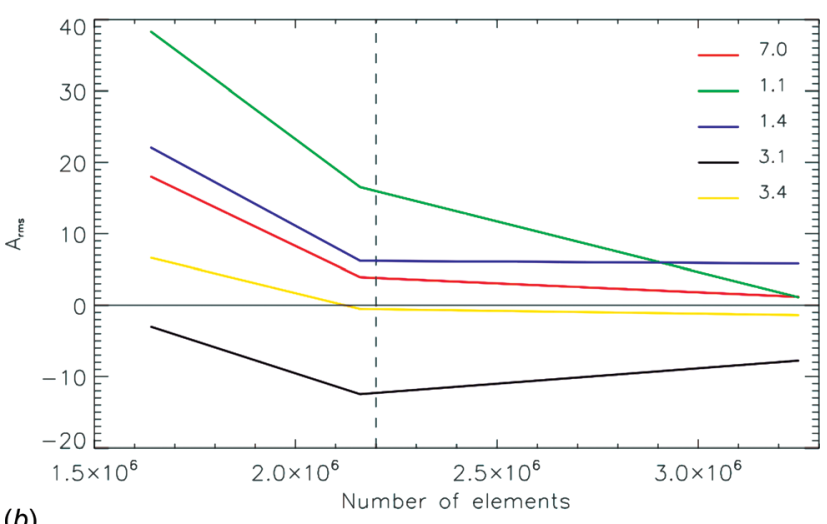

Fig. 6 Error in mass flow rate for the five geometries: (a) supply port $\left(W_{s}\right) ;(b)$ outlet port $\left(W_{o}\right)$

radial and tangential flows in the vortex chamber proper (Sec. 6.2); the precessing vortex core in the outlet (Sec. 6.3); and the back-flow in the supply ports (Sec. 6.4). Finally, the comparative performance of the five selected supply port geometries is considered (6.5).

6.1 Validation of the Simulations Against the Experimental Data for the Five Selected Supply Port Geometries. Simulations were carried out on the subset of five geometries $(2.2,2.3,3.2$, 3.3 , and 7.0) selected by the air experiments in Sec. 2. The geometry, mesh, and initial physical boundary conditions described in Sec. 4 were used to generate a definition file containing the differential equations of mass, momentum, and energy conservation applicable to the problem. This definition file is then used as input for the CFX solver which determines a numerical solution to the differential equations. The solver creates a results file which can be viewed using the CFX postprocessing software.

The results of the validation exercise for all five selected geometries are shown in Fig. 7, in which the simulated and experimental mass flow rates at both the supply and outlet ports are compared against the simulated rates at the control ports (it was not possible to measure the flow rates at the control ports). The average error over the range of geometries tested was found to be in the region of $10.5 \%$, the maximum being $16.6 \%$ for geometry 7.0. Given the errors identified by the mesh verification exercise (from $3.6 \%$ to $8 \%$ ) and the levels of uncertainty associated with the test rig equipment (i.e., gas transmitter $\pm 2 \%$, digital pressure transducer $\pm 1.5 \%$ ), it is considered that the simulation of the VXA provides a sufficiently accurate representation of the experimental results.

6.2 Radial and Tangential Flows in the Vortex Chamber Region. The radial and tangential velocity profiles for geometry 7.0 can be seen in Fig. 8. Though an exhaustive comparison of simulated and experimental data was not possible because the air and smoke tests were unable to provide comprehensive experimental data, the flow structures simulated within the VXA accord well with previously published data; radial and tangential velocity profiles qualitatively match those produced by Syred and Royle [17] and Savino and Keshock [18] using pitot-yaw probes.

Figure $8(b)$ shows how the majority of radial flow passes close to the end walls of the vortex chamber, the flow increasing axially in velocity as it approaches the chamber walls before succumbing to nonslip retarding effects. The spacing of the lines also indicates that the radial flow accelerates the closer it gets to the exit port outlet. At the periphery of the chamber $(x=65 \mathrm{~mm}$ to $x=55 \mathrm{~mm}$ ) the radial flow profile is fairly even. However, toward the outlet it is reversed from the mid-plane of the chamber $(h=4 \mathrm{~mm}$ to $h=8 \mathrm{~mm}$ for $P_{c}=-374 \mathrm{~Pa}$ ) out toward the supply and control ports. As the value of $P_{c}$ is increased the area of reversed radial flow also increases.

The velocity profiles in Fig. 8(c) indicate that the majority of the tangential flow is contained away from the walls of the chamber, the tangential velocity increasing significantly from $x=30$ $\mathrm{mm}$ to $x=15 \mathrm{~mm}$. The anomalous tangential profile for $\mathrm{x}=65$ $\mathrm{mm}$ is a result of the location of the sample line with respect to the supply port, the line being located at the point where flow from the supply port meets the spinning vortex. Across the height of the chamber the distribution of tangential velocity is fairly even, falling away sharply towards the chamber walls. As tangential velocity increases towards the exit port the radial velocity in the center plane of the vortex can be seen to fall away slightly. Explanations of the forces that influence tangential and radial velocity vectors in short vortex chambers (i.e., $h / r_{e}<2$ ) have been published previously [4,17-21].

The profiles for $P_{c}=-374 \mathrm{~Pa}$ in Fig. $8(\mathrm{c})$ show that the tangential velocity increases as the radius within the vortex chamber decreases, the majority of the chamber being filled with non-rotational flow. In Fig. 8(c) it is also worth noting that, for the same value of $P_{c}$, radial flow exists across the full height of the chamber from $x=65 \mathrm{~mm}$ to $x=30 \mathrm{~mm}$. Both tangential and radial flow profiles from $x=65 \mathrm{~mm}$ to $x=50 \mathrm{~mm}$ are within the mixing region of the supply and control port inlets and show developing flow profiles. The ideal flow structure for maximum pressure drop across a vortex chamber is one where a free vortex structure exists across the full height of the chamber [22], the radial flow having to make its way across the chamber against the strong centrifugal forces generated by the free vortices. The swirl number for the device varied from 3.4 to 5.9 across the range of simulated pressures.

As the magnitude of $P_{c}$ increased, the tangential velocity in the outer portion of the vortex from $x=50 \mathrm{~mm}$ to $x=30 \mathrm{~mm}$ decreased towards the vortex core, indicating the development of a forced vortex. The rotational system is effectively the mixing area generated by the tangential control port inlets. The width of the rotational system is a function of the VXA geometry: the wider the mixing region for a given value of $r_{o}$ the less efficient is the device at creating a vortex. A similar structure was identified by Syred [23] and MacGregor and Syred [24], the size of the forced rotational flow area occupying nearly a third of the width of the chamber (i.e., a forced vortex mixing region at $r / r_{e}>0.67$, with a free vortex region at $\left.r / r_{e}<0.67\right)$. These values were based upon an average swirl number of 28.94. Once established, the actual width of the peripheral forced vortex changed only slightly over the range of simulated pressures.

From $x=30 \mathrm{~mm}$ to $x=15 \mathrm{~mm}$ the chamber is filled with a free vortex flow; the overall vortex generates sufficient centrifugal force to balance the inward radial pressure gradient toward the chamber walls, creating a reverse flow in the mid-plane of the chamber. The parabolic radial velocity profiles are a consequence of interaction between the vortex and the chamber walls. As viscous effects reduce tangential velocity at the walls so the centrifugal field $\left(m v^{2} r\right)$ generated by the tangential flow weakens (by the square of the tangential velocity) and is overcome by the radial pressure field. Radial flow is drawn through the circumferential boundary layers, accelerating as the free area reduces towards the 

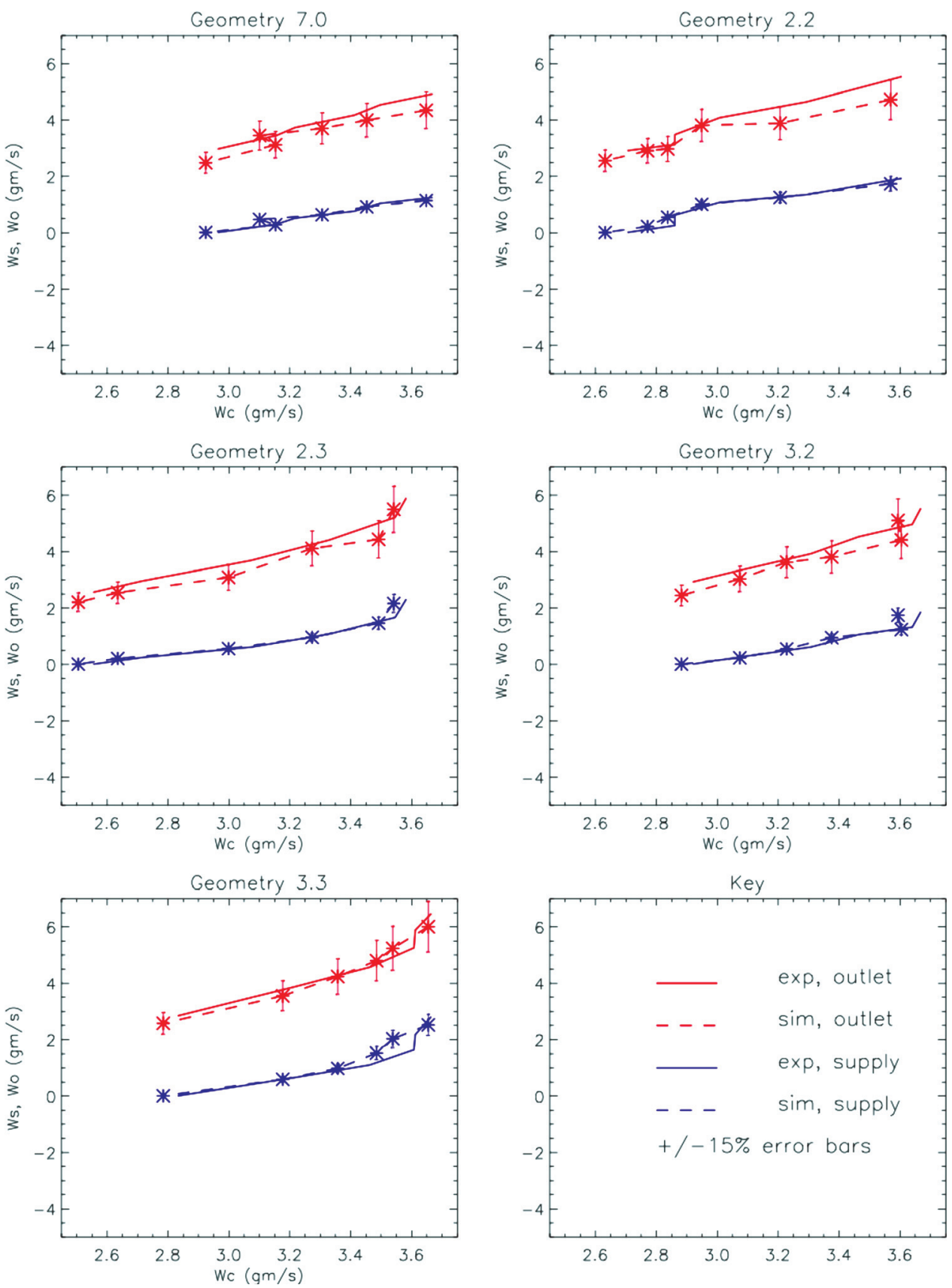

Fig. 7 Comparison of experimental (solid) and simulated (dashed) results, showing mass flow rates for both supply (blue) and outlet (red) against simulated control port mass flow rates. Error bars with $a \pm 15 \%$ range have been included to give an indication of the margin of error between the experimental and simulated results.

center of the chamber. The proportion of flow that moves through the boundary layers is a function of swirl. As the value of $P_{c}$ is increased and the strength of the mid-plane reversed radial flow increases as a result, so the incoming radial flow through the supply inlets is forced into the boundary layers before leaving the supply ports. At the center of the chamber, where the flow finally reaches the axial outlet, the flow regime again returns to a forced vortex form to avoid the creation of a singularity at the vortex core (i.e., $V r^{n}=$ constant at $r=0$ ). The greatest pressure losses occur at the entrance to the exit port where the highly turbulent flow leaves the chamber.

6.3 Exit Port Region. Such is the angular velocity of the flow leaving the chamber that the radial pressure field generated to balance the centrifugal force creates a region of negative static pressure at the center of the vortex core (usually about the central axis of the outlet diffuser). Similar to flow through the vortex chamber, the majority of the axial flow travels down the sides of the exit port walls in the boundary layer regions (Fig. 9). The vortex core is clearly established in the central pipe region, with most outflow along the pipe wall. An off-center vortex core was also produced by Woolhouse et al. [6]. What remains in question is whether the lack of symmetry in Fig. 9 is a numerical (or mesh) effect, or convergence on one possible solution, to imply (in this steady state solution) the capture of evidence of vortex precession. Monitoring of convergence during the current study has revealed that it is the very low velocity regions in the glovebox that remained slow to converge.

Recirculating flows have been observed previously $[24,25]$ and are known to exist over a range of Reynolds numbers $\left(R_{e}\right)$ from 
VXA chamber

Outlet

(a)
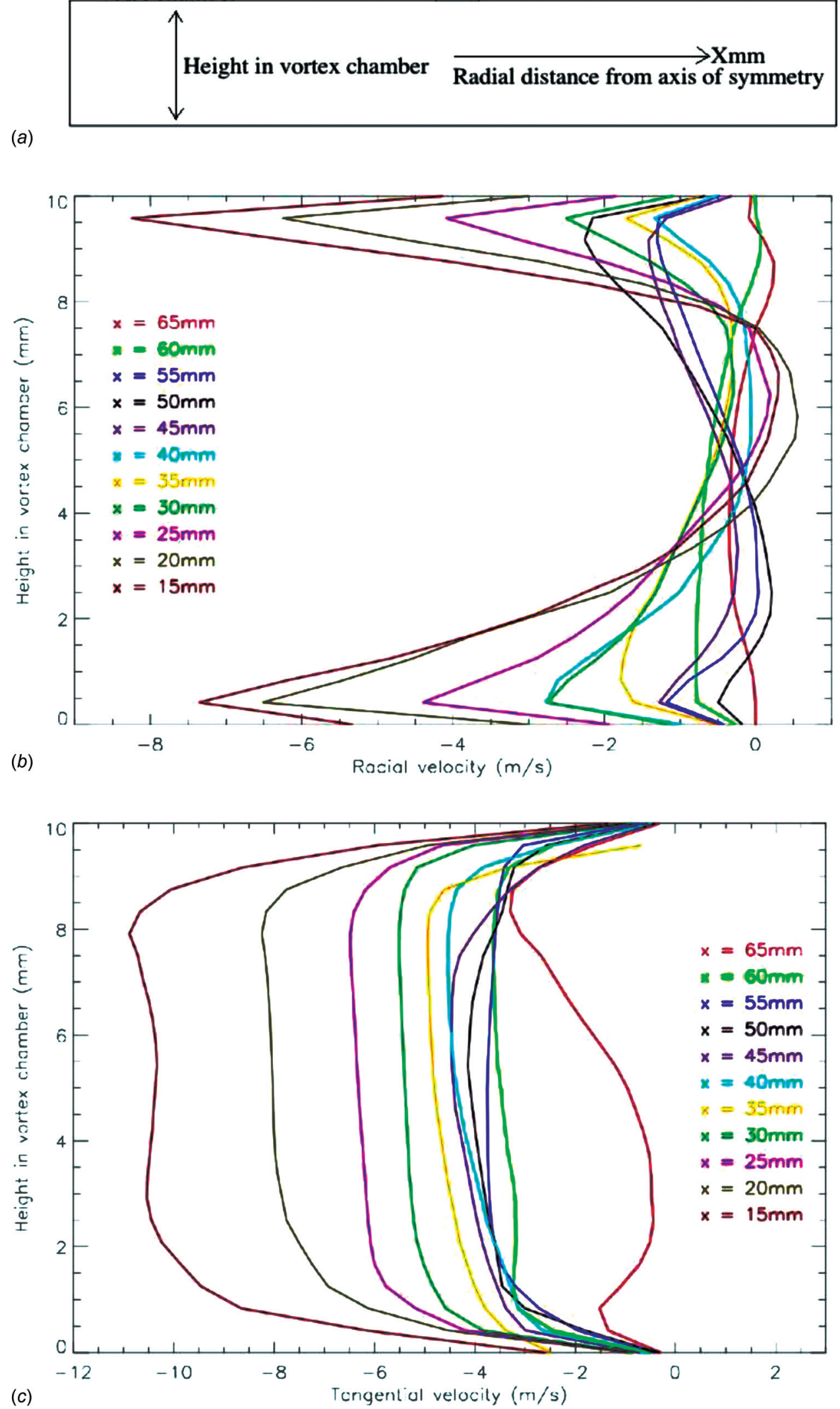

Fig. 8 Geometry 7.0 and $P_{c}=-374$ Pa: (a) cross-section of VXA chamber showing radial distance from axis of symmetry $(x \mathrm{~mm})$, and height in vortex chamber; $(b)$ radial velocity profiles; (c) tangential velocity profiles 


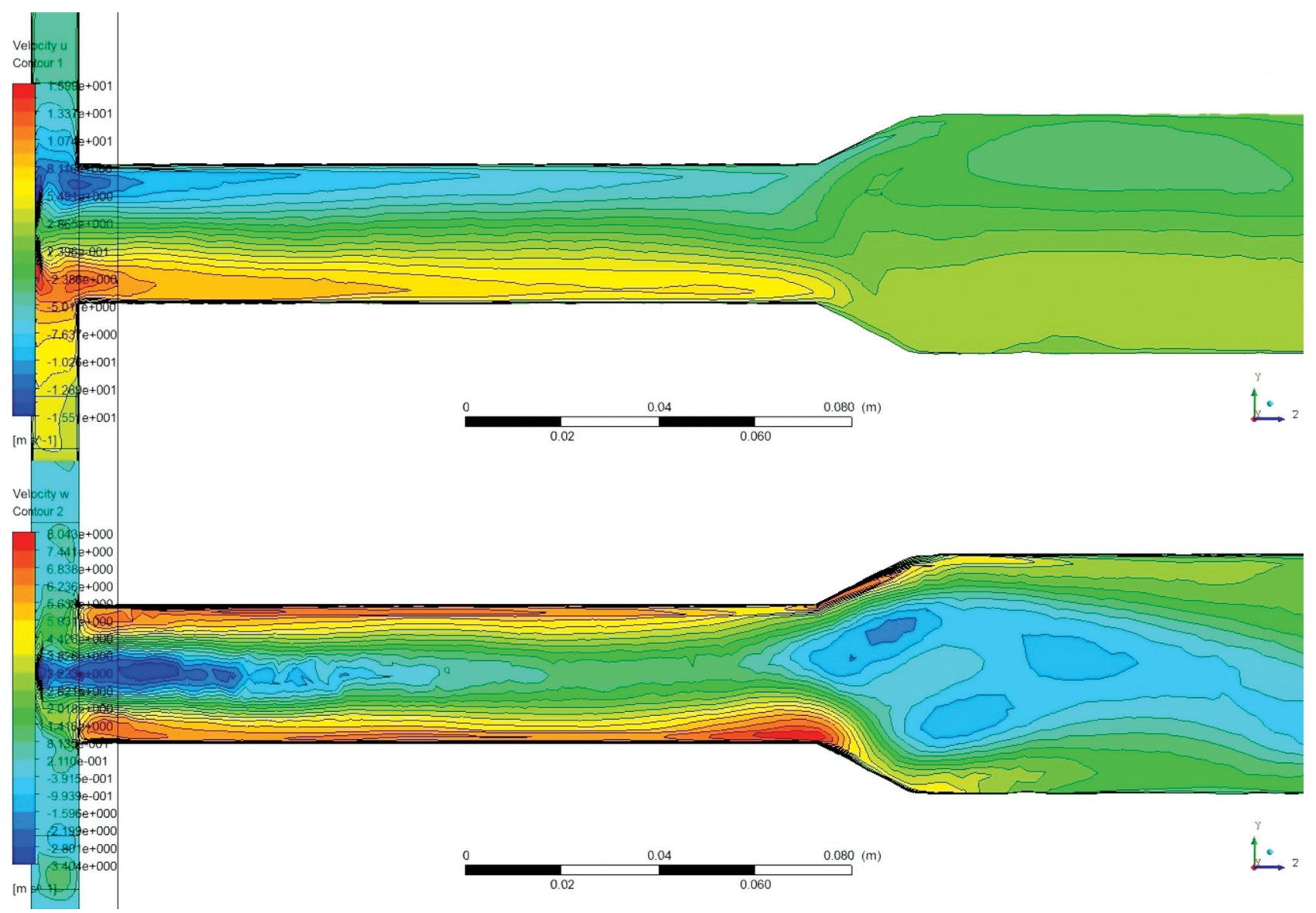

Fig. 9 The vortex core in the VXA outlet

$1 \times 10^{3}$ to $1 \times 10^{4}$. The actual point at which the zone of reverse flow occurs has been linked to the swirl factor $(S)$. Over the range of simulations conducted with geometry 7.0, values of $R_{e}$ in the exit port vary from 5459 to 5819 , with $S$ ranging from 3.4 to 5.9 . These values are similar to those associated with axial flow reversal and the formation of precessing vortex cores, which are symptomatic of nonaxisymmetric fluid flow in the VXA.

The velocity profiles about the centre of the axial outlet suggest that flow is axisymmetric. However, closer study of flows at the walls of the exit port duct show an uneven axial velocity profile (Fig. 10). At $z=-20 \mathrm{~mm}$ axial flow away from the chamber is slightly faster on the left-hand side of the exit duct, the actual area of fast axial flow $(w<3.81 \mathrm{~m} / \mathrm{s})$ being slightly greater than that on the right-hand side. The same effect can be seen at each of the sample points, the fastest axial flows swapping from left to right at $z=-40 \mathrm{~mm}$ and then back again at $z=-100 \mathrm{~mm}$.

It is possible that the uneven axial velocity profiles are the result of a precessing vortex core. As stated above, such structures have been observed experimentally at similar values of $R e$ and $S$ and, once generated, a precessing vortex core would disrupt axial flow at the points where the helical structure comes close to the walls of the outlet. Figure 10 gives only a general impression of the spatial variation in axial velocity; visualization of such structures is best achieved using transient simulation techniques (to be published in an accompanying paper).

6.4 Supply Port Region. Further evidence of asymmetrical fluid flow can be found at the supply port inlets. Again focussing on geometry 7.0, Fig. 11 gives both the cumulative $\left(W_{s}\right)$ and individual $\left(W_{s}\left(S P_{n}\right)\right)$ mass flow rates in the supply ports for control port pressure from -374 to $-370 \mathrm{~Pa}$. Positive values indicate flow into the VXA supply ports, negative values indicate leakage into the glovebox. At $P_{c}=-374 \mathrm{~Pa}$ both the cumulative supply flow $\left(W_{s}\right)$ and the mass supply flow through each of the supply ports is positive. As the value of $P_{c}$ is increased to $-373.5 \mathrm{~Pa}$ the values of $W_{s}(S P 1)$ and $W_{s}(S P 4)$ become negative, while $\left(W_{s}\right)$ is reduced by a factor of about 200 but remains positive. As the value of $P_{c}$ is further increased $W_{s}$ becomes negative, although at $P_{c}=-373 \mathrm{~Pa}$ flow through $W_{s}(S P 2)$ and $W_{s}(S P 4)$ is still positive. At no point in the simulation exercise for geometry 7.0 could flow through the supply ports be described as axisymmetric.

Instabilities in the VXA's flow regime were noted during the smoke visualization investigations. The results in Fig. 11 indicate that the mechanism for back-flow diffusion at the supply ports may be even more subtle than first thought. Standard modeling practice for multiple control and supply port devices assumes axisymmetric flow. However, instabilities are known to exist, even in the most apparently stable devices. If flow within the VXA at high levels of swirl is asymmetric it is possible that, while maintaining an overall positive value of $W_{s}$, mass flow through the supply ports may fluctuate from positive to negative.

At $P_{s}=-373 \mathrm{~Pa}$ flow through the supply port inlets was slightly negative $\left(W_{s}=-7.199 \times 10^{-5} \mathrm{~kg} / \mathrm{s}\right)$. The velocity vector plots in Fig. 12 clearly show the uneven flow structures formed at each of the supply inlets. The effect of inflated prismatic cells at the walls of the inlet can be seen in each plot, the flow being subject to the nonslip condition set at the walls. Despite the geometric symmetry of the VXA, the asymmetric location of the vortex at the supply port inlets is clear in $S P 1, S P 3$, and SP4. In SP2 the edge of the vortex touches the left side of the supply port wall; supply flow is entrained by the much faster vortex flow. Propelled towards the supply port wall on the left, the flow is then turned sharply away from the central vortex forming a large area of 


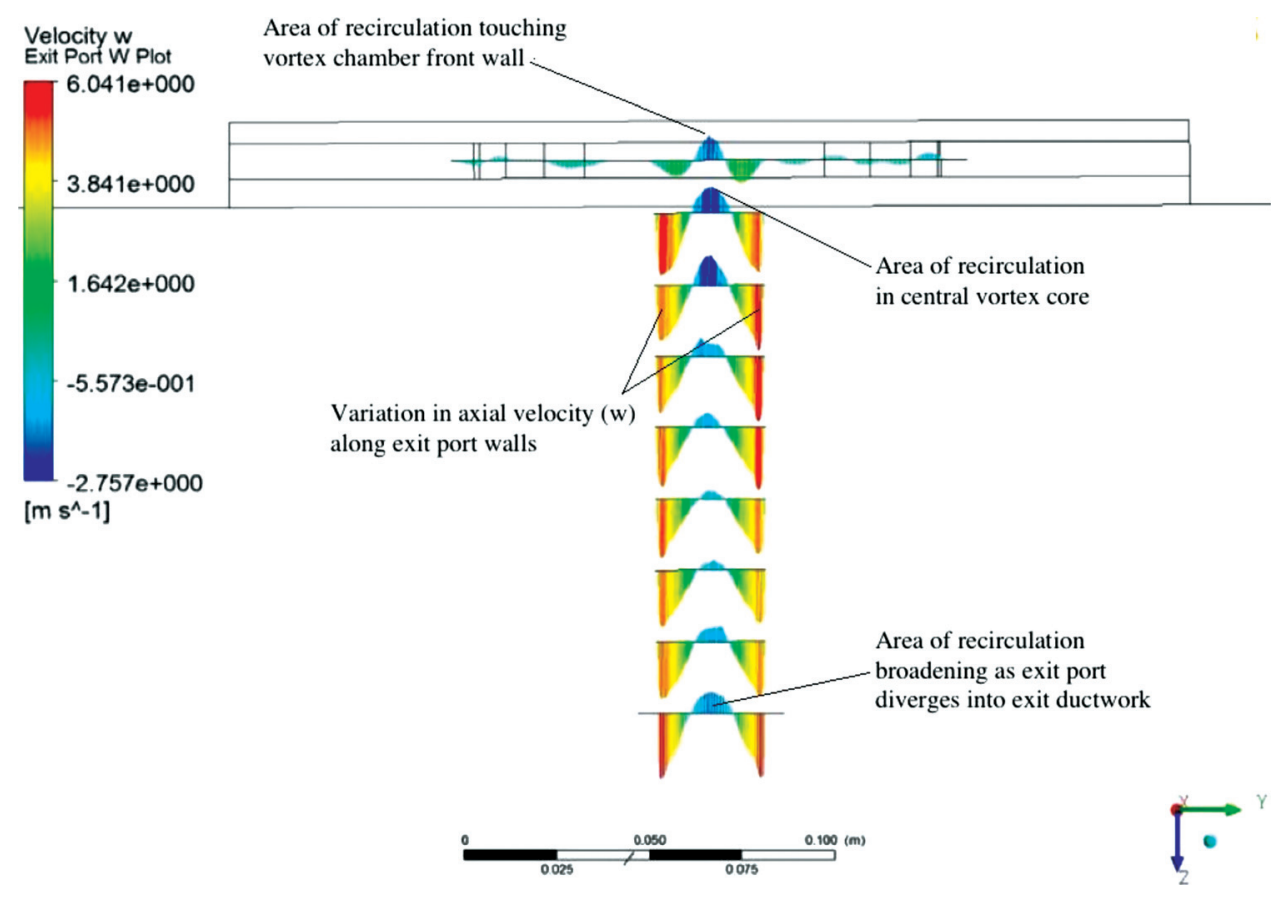

Fig. 10 Axial (z) velocity vector profiles for geometry $7.0\left(P_{c}=-373 \mathrm{~Pa}\right)$

recirculation within the supply port inlet. Despite the area of recirculation, flow through $S P 2$ is still positive.

In SPI a clear area of leakage can be seen leaving the supply port along the left-hand wall; this is despite there being a gap between the supply port wall and the edge of the vortex. An area of recirculation can be seen in the top left corner of the supply port inlet; flow entering this area is turned sharply at the supply port wall and forced back out the supply port inlet. A similar flow regime exists in $S P 3$, with flow leaving the supply port along the left-hand wall. However, unlike $S P I$ no area of recirculation has developed within the supply port, which is probably due to the reduced gap between the edge of the vortex and the supply port wall. In both $S P 3$ and $S P 1$ supply flow was reversed.

In $S P 4$ the gap between the vortex and the chamber wall is significant, possibly the largest of all four supply ports. Flow through the port is positive; however, there are indications of an area of recirculation starting to form similar to that in SP1. This could explain why

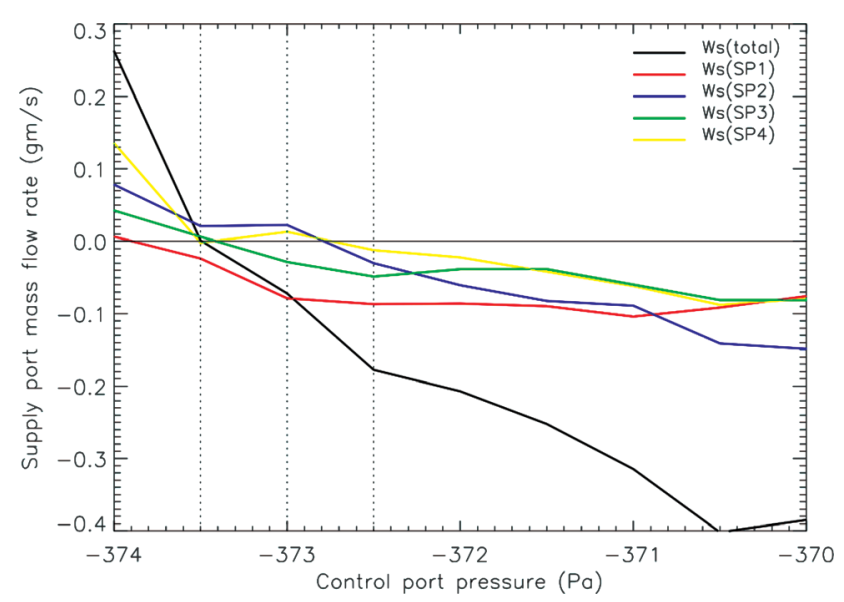

Fig. 11 Geometry 7.0 supply port mass flow rates for a range of control port pressures from -374 to $-370 \mathrm{~Pa}$ (back-flow when mass flow rate $<0$ ) mass flow rates are still below those through $S P 2$, despite having a large area for entrained flow to pass into the vortex chamber.

Results of the smoke tests for all the geometries clearly show asymmetric flow distribution in the VXA supply ports and what appears to be a time-dependant instability at the vortex core. Results of the simulation indicate the presence of an asymmetric vortex flow (possibly a precessing vortex) in the exit port duct and an area of reverse flow inside the vortex core. The reverse flow can be seen to pass through the throat of the exit port, attaching itself to the back-wall of the vortex chamber. Such flows are known to be unstable. As the flow in one region of the VXA has a direct effect on the regions adjoining it, it is reasonable to assume that disturbances at the control and supply inlets caused by the asymmetric supply port flows could be transmitted downstream through the outlet vortex by a feedback process, creating further disturbances. While values for supply flow are low (from approximately $2.628 \times 10^{-4} \mathrm{~kg} / \mathrm{s}$ to $3.848 \times 10^{-4} \mathrm{~kg} / \mathrm{s}$ for geometry 7.0), even a slight perturbation in pressure at the periphery of the vortex would be amplified, potentially resulting in significant feedback.

Typical nitrogen purge rates through a Mini-VXA range from $2.60 \times 10^{-4} \mathrm{~kg} / \mathrm{s}$ to $2.13 \times 10^{-4} \mathrm{~kg} / \mathrm{s}$, similar to the supply flow rates found in the simulations. While VXAs with radial diffusers are known to be more stable than those with conical or straight edges, perturbations in pressure and flow (i.e., noise) have still been found and recorded [22-24]. It is therefore likely that the same mechanisms responsible for back diffusion of control flow in the simulated VXA geometries are responsible for leakage in Mini-VXAs.

While purge flow rates through gloveboxes are fixed, it is possible that time-dependant flow regimes in the vortex core of the Mini-VXAs and instabilities in the control port flow create a situation where asymmetric flow exists between the supply ports. As seen from the results of the geometry 7.0 simulations, asymmetric flow can cause one or more of the supply ports to pass control flow into the glovebox whilst the other supply ports act in a positive direction. The aggregate mass flow rate can still remain positive with respect to the VXA, the negative leakage through one supply port being offset by increased mass flow through one or more of the others. A flow regime such as this would cause 


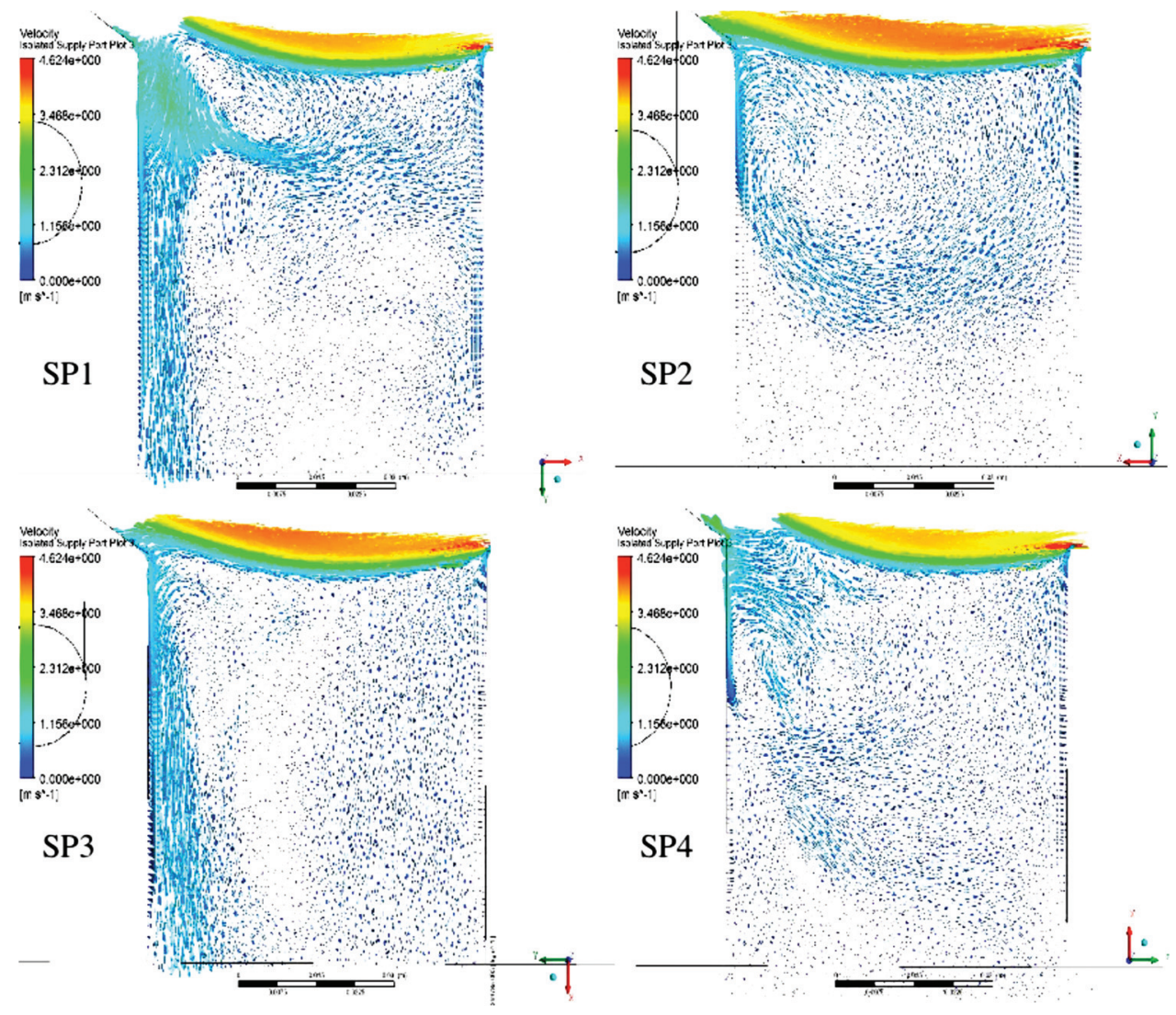

Fig. 12 Geometry 7.0 supply port velocity vector plots $\left(P_{s}=-373 \mathrm{~Pa} ; z=-5 \mathrm{~mm} ; W_{s} \mathrm{~kg} /\right.$ $s \times 10^{-5}$ : SP1 7.870; SP2 2.227; SP3 2.850; SP4 1.294; see Fig. 3 for layout of supply ports SP1-4 around the vortex chamber)

oxygen levels in the glovebox to rise while still being subject to a positive purge flow.

Given that most radial VXAs have a similar control and supply port design it is likely that the potential for asymmetric supply flow exists in all of them. However, in standard ventilated enclosures using air, back diffusion would not be noticed. Furthermore, previous VXA incarnations have supplementary geometric features that might have masked the oxygen diffusion issue. For example, the VXAs were fitted in-line, downstream of the glovebox, or fitted with filters over the supply ports [2]. Given the above, it is understandable that the back diffusion problem did not come to light

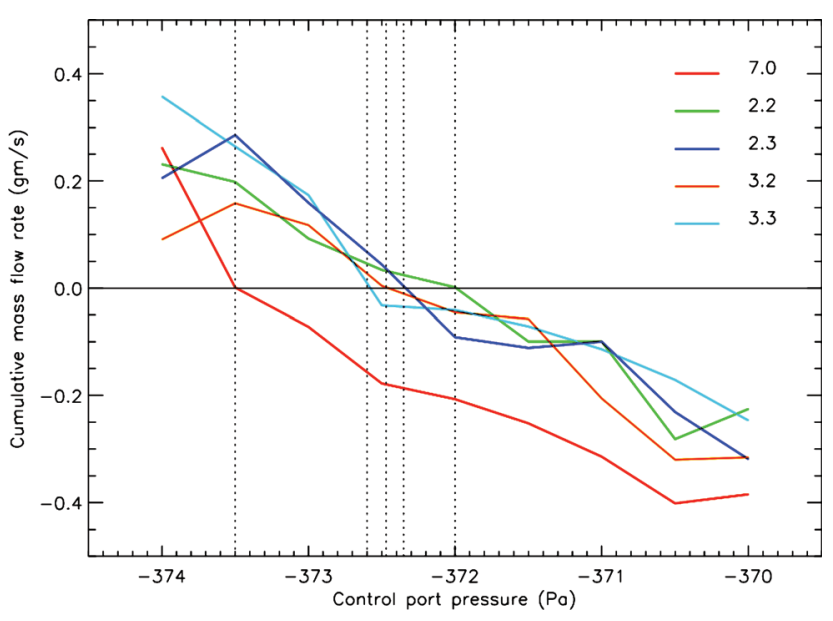

Fig .13 $W_{s}$ against $P_{c}$ for geometries 2.2, 2.3, 3.2, 3.3, and 7.0 until after the development of the Mini-VXA and its subsequent use on purged gloveboxes.

6.5 Comparative Performance of the Five Supply Port Geometries. Because the primary driving mechanism for the leakage appears to be instabilities in the vortex core and control port flow, it is unlikely that the fundamental problem can be resolved without significantly redesigning the Mini-VXA units. However, the results from the smoke experiments indicate that it is possible to at least delay the onset of leakage by slightly amending the supply port geometry to introduce an opposing tangential element into the supply flow.

Performance of the alternative supply port geometries was assessed against their ability to prevent or reduce leakage from the VXA for a given value of $P_{c}$. The results of the simulations have been plotted in Fig. 13, which shows aggregate mass flow rate $\left(W_{s}\right)$ against $P_{c}$. While aggregate flow rates provide a reasonable indication of the performance of a VXA, they do not capture the effects of simultaneous flow in and out of a single supply port; nor do they capture the effects of negative flow through one or more of the supply ports (the aggregate still remaining positive). At best, aggregate leakage can be considered to be a crude measure of performance in this context. However, it reasonable to assume that if the alternative geometries can delay the point at which aggregate flow through the supply ports becomes negative they must also reduce or delay the other more transient forms of leakage.

In Fig. 13, the red line for geometry 7.0 lies below that of the other geometries (with the exception of the point at $P_{c}=-374$ $\mathrm{Pa}$ ), crossing the $\mathrm{x}$-axis into negative flow at about $P_{c}=-373.5$ $\mathrm{Pa}$. The alternative geometries $(2.2,2.3,3.2$, and 3.3) do not cross the x-axis until after $P_{c}=-372.6 \mathrm{~Pa}$ at which point they start to diverge slightly. From the graph it is clear that the alternative 


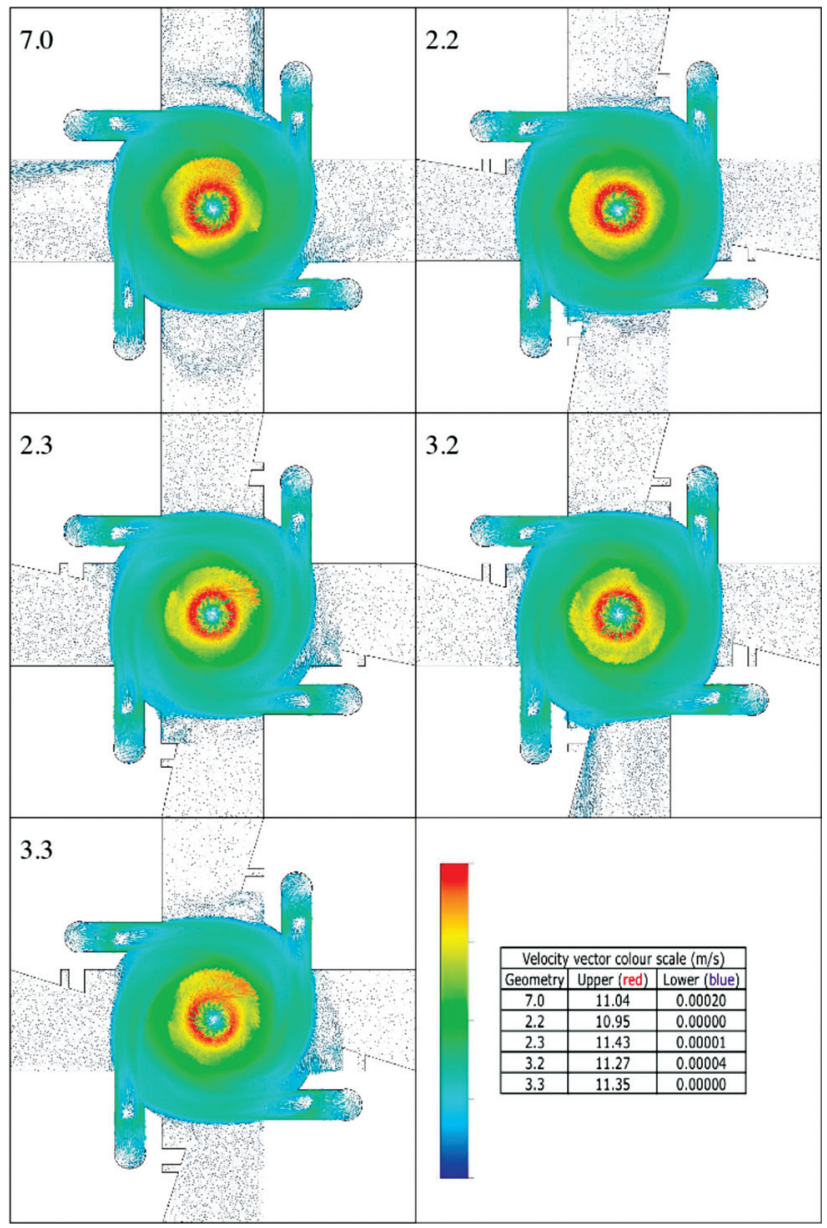

Fig. 14 Velocity vector plots for geometries 7.0, 2.2, 2.3, 3.2, and 3.3, based on a control port pressure of $-373 \mathrm{~Pa}$

geometries delay the onset of back-flow and continue to mitigate it over the range of pressures simulated.

Although not clear on the graph, the geometry with the best performance was 2.2, and the worst was 3.3, despite the fact that geometry 2.2 showed reverse flow through one of its supply ports $(S P 2)$ at a slightly lower pressure than two of the other three alternative geometries. The aggregate mass flow rate through geometry 2.2 did not reverse until $P_{c}=-372.0 \mathrm{~Pa}$, unlike geometries 2.3 and 3.2, which succumbed to reverse flow slightly above $P_{c}=-372.5 \mathrm{~Pa}$. Supply flow through geometry 3.3 reversed at $P_{c}=-372.6 \mathrm{~Pa}$. All four alternative geometries show a significant improvement on geometry 7.0.

'A comparison of the velocity vectors for the five simulated geometries can be seen in Fig. 14, based on a control port pressure of $P_{c}=-373 \mathrm{~Pa}$. At this point aggregate supply flow through geometry 7.0 was negative, while flow through the other four geometries remained positive. Table 5 shows the calculated mass flow rates through each of the supply ports for the five geometries in turn.
By comparing the vector plots in Fig. 14 in relation to Table 5 it is clear that in geometry 7.0 the flow has been reversed through $S P 1$ and $S P 3$, the densest patch of vector lines to the right of the $S P 1$ supply port indicating the area of greatest leakage. An area of recirculation can be seen just starting to form between the backflow and the edge of the vortex. Similar areas of recirculation appear to be forming in SP2 and SP4, just prior to the switch from positive to negative flow.

In geometry 2.2 small areas of recirculation have formed in the square areas created by the top of the converging taper and the supply port wall, the most significant being that in $S P I$. Flow through $S P 2$ has been reversed. However, the path taken by the reverse flow is not clear in the picture; what is clear is the small area of turbulent flow situated where areas of recirculation exist in the other supply inlets. Actual tangential velocity has also been reduced both at the boundaries of the vortex in the forced vortex region and further on, in the free vortex area.

In geometry 2.3 the flow is positive through all four supply ports. The effect of the increase in $y$ from $5 \mathrm{~mm}$ to $10 \mathrm{~mm}$ is clear, the areas of recirculation being very prominent in $S P 4$ and $S P 2$. It is interesting to note that in both supply ports the area of recirculation appears to push the tangential jet stream away from the supply port wall, creating a gap between the edge of the vortex and the end of the supply inlet. Despite the noticeable gap, mass flow through these ports is significantly lower than that of the other supply ports (SPI and SP3). This same phenomena can be seen in the vector plot for geometry 3.3, where the area of recirculation formed in SP4 (and to a lesser extent SPI) seem to push the tangential stream away from the walls. Again both supply inlets show significantly less positive mass flow than the other inlets.

In geometry 3.2 a clear area of reverse flow can be seen leaving the VXA via SP2 along the edge of the converging taper. Above the taper is what appears to be a large area of tangential flow bifurcating as it meets the edge of the taper. The other supply ports show some initial signs of recirculation starting to form at the top of the tapers, the most significant being that for SP3. Again, there are signs of the tangential flow being diverted.

All four geometries showed a reduction in tangential velocity across the vortex caused partly by the opposing tangential supply flow generated by the diverging tapers, and partly by the recirculation areas formed between the top of the tapers and the vortex. It was originally envisaged that the areas of recirculation were formed through bifurcation of the control flow stream at the supply wall. However, it would appear that when operating the VXA under these conditions the areas of recirculation are formed by entrained supply flow which then acts to move the control port jet stream away from the supply port wall.

\section{Discussion}

From the results it has been possible to identify the most promising prototype geometry and, by means of laboratory tests and field trials, to demonstrate its efficacy at substantially reducing inert gas usage (to be reported in an accompanying paper). This paper focuses on the steady-state computational aspects of the investigation. Subsequent work then diverged with prototype testing taking place alongside an academic study of periodic effects in a transient CFD study.

Table 5 Geometry 7.0, 2.2, 2.3, 3.2, and 3.3 mass flow rates (for $P_{c}=-373 \mathrm{~Pa}$ )

\begin{tabular}{|c|c|c|c|c|c|}
\hline \multirow[b]{2}{*}{ Geometry } & \multicolumn{5}{|c|}{$P_{c}=-373 \mathbf{P a}$} \\
\hline & $W_{s}$ & $W_{s}(S P 1)$ & $W_{s}(S P 2)$ & $W_{s}(S P 3)$ & $W_{s}(S P 4)$ \\
\hline 7.0 & $-7.20 \mathrm{E}-05$ & $-7.87 \mathrm{E}-05$ & $2.23 \mathrm{E}-05$ & $-2.85 \mathrm{E}-05$ & $1.29 \mathrm{E}-05$ \\
\hline 2.2 & $9.24 \mathrm{E}-05$ & $3.70 \mathrm{E}-05$ & $-3.74 \mathrm{E}-05$ & $5.76 \mathrm{E}-05$ & $3.51 \mathrm{E}-05$ \\
\hline 2.3 & $1.59 \mathrm{E}-04$ & $4.88 \mathrm{E}-05$ & $2.61 \mathrm{E}-05$ & $6.35 \mathrm{E}-05$ & $2.10 \mathrm{E}-05$ \\
\hline 3.2 & $4.10 \mathrm{E}-05$ & $3.73 \mathrm{E}-05$ & $-3.83 \mathrm{E}-05$ & $3.499 \mathrm{E}-05$ & $4.20 \mathrm{E}-05$ \\
\hline 3.3 & $1.74 \mathrm{E}-04$ & $1.43 \mathrm{E}-05$ & $7.10 \mathrm{E}-05$ & $7.10 \mathrm{E}-05$ & $1.75 \mathrm{E}-05$ \\
\hline
\end{tabular}


The results indicate a preferred geometry, one in which the taper is foreshortened by such a length that a recirculating zone is created for the bifurcated control port jet, redirecting the flow that would otherwise propagate along that edge of the port back into the glovebox. The experiments conducted for characterization of the vortex amplifier (not reported here) demonstrate that, excepting one point on the characteristic curve well away from the normal operating point, the average/global mass flow and pressure readings are stable; hence the periodic effects of a precessing vortex can be neglected for the purpose of the study. Nevertheless, the results demonstrate asymmetry, which is worthy of further consideration.

The VXA chamber itself contains substantial internal curvature, producing swirl and high pressure gradients along radii. It is impossible to select a turbulence model, wall functions, discretization method, and solution algorithm that is favorable to all elements of the problem. Nevertheless, use of the BSL RSM produced flow structures that are known to exist in the practical geometry at equivalent swirl and Reynolds numbers, including something akin to the spinning doughnut and in particular, the vortex core in the outlet diffuser. The question remains as to whether the asymmetry demonstrated in the solution was the result of a numerical (or mesh) effect, or convergence on one solution from a range of possible solutions that would try to capture a precessing vortex core.

The use of unstructured meshes with large scale ranges leads to mesh structures that are not identical in all four supply ports. Moreover, convergence is often very slow after an initial 250 iterations, due to problems in the wider domain of the glovebox where flow velocities are extremely low (the residuals being smaller in the VXA). This might be the result of using a multigrid approach.

There are two surface meshing routines available in the CFXMESH package. Although slower than the Delaunay Surface Mesher, the Advancing Front Surface Mesher was purported to produce a better quality mesh on poorly parameterized surfaces. Unfortunately, the Advancing Front Surface Mesher cannot generate mesh on a closed surface. While parameterization was an issue with the VXA geometry, the closed faces formed by the exit diffuser, extract ductwork, and control port inlets meant that the Advancing Front Surface Mesher could not be used.

Element volume ratios, node connectivity, and orthogonal quality measures were easily achieved in the bulk of the domain, but in the near-wall regions within the VXA it proved impossible to maintain that quality. Element volume ratios were most difficult to achieve for the prisms and pyramids, the types of elements used in the generation of the inflated boundary layers. In many instances the mesh had to be further refined to reduce their number. It was not possible to completely remove these types of elements that exceeded the recommended ratios because regeneration simply produced new skewed elements in different areas of the mesh. Care was taken when specifying the mesh length scales to ensure that the expansion factor remained at no more than 1.2. By this means, large variations in coincident element size and length were avoided. Likewise, a few nodes experienced connectivity figures in excess of the 12 recommended for prisms. Also in this region, a few tetrahedral elements where identified in each model with face angles below $10^{\circ}$, the problem areas being the corners of the walls inside the chamber where two inflated boundary layers meet. There was little that could be done to improve these areas without generating a prohibitively overrefined mesh.

\section{Conclusions}

(1) It has been verified that there exits a mesh length scale limit beyond which further simulation accuracy cannot be achieved. In the case of the Mini-VXA the maximum residual values for momentum $\left(U_{\text {mom }}, V_{\text {mom, }} W_{\text {mom }}\right)$ and mass $\left(P_{\text {mass }}\right)$ were $1 \times 10^{-4}$ which occurred when the number of mesh elements in the model approached $2 \times 10^{6}$.
(2) Comparison of the simulated and experimental results for the five selected geometries reveals that it is possible to model global mass flow through the VXA with specified boundary pressures to an average error of $10.5 \%$.

(3) The model was able to reveal known critical flow structures, including a vortex core and a control port jet which bifurcates after impinging on the opposite supply port wall. The model was useful in determining the most promising prototype geometry for maximum reduction of inert gas usage.

(4) The prototype supply port geometry using a taper reduces the magnitude of reverse flow through the supply ports. Of the alternative prototypes considered, geometry 2.2 gave the most effective reduction of reverse flow through the supply ports.

(5) The simulations show transient eddies in the supply port outlet with reversed flow in certain supply ports, while the aggregate flow through the device remains positive. There is significant asymmetry between the four supply ports.

(6) The effects of asymmetric flow in the supply ports is unlikely to have been recognized in earlier VXAs used by British Nuclear Fuels Ltd. because the actual operation of the VXA (or its location in relation to the glovebox) masked the oxygen back-flow leakage effect.

(7) It has been shown that there is evidence of a precessing vortex core in the outlet. The mutual asymmetry between the four supply port flow structures is related to the procession of the vortex core. This work demands a transient CFD study to establish the veracity of the proposed relation, and to reveal any causal link.

(8) Convergence is a relative concept when simulating flow at laminar and turbulent scales, and only loose convergence can be expected when including in the flow domain the large reservoirs surrounding the main internal flow component of interest.

(9) Use of a baseline Reynolds Stress Model incorporating additional hybrid functions for thin sections has enabled the capture of anisotropic stress and strain that would be expected from observations of real flows.

Although only reported fully in the accompanying "transient" paper, it should be noted herein that the efficacy of the preferred geometry 2.2 was subsequently confirmed in field trials at the Nexia BTC facility.

\section{References}

[1] Wormley, D. N., and Richardson, H. H., 1970, "A Design Basis for VortexType Fluid Amplifiers Operating in the Incompressible Flow Regime," J. Basic Eng., 92, pp. 369-376.

[2] Birch, M. J., Doig, R., Francis, J., Parker, D., and Zhang, G., 2009, "A Review of Vortex Amplifier Design in the Context of Sellafield Nuclear Operations," Proceedings of the $12^{\text {th }}$ International Conference on Environmental Remediation and Radioactive Waste Management, October 11-15, Liverpool, UK ASME, New York, Paper No. 16063.

[3] Shimizu, A., Matsumoto, Y., and Wada, T., 1985, "Numerical Study of Swirling Flow in a Vortex Device," Proceedings of the Conference on Fluid Control and Measurement, IOS Press, Amsterdam, pp. 353-358.

[4] Wormley, D. N., 1969, "An Analytical Model for the Incompressible Flow in Short Vortex Chambers,” J. Basic Eng., 91, pp. 264-276.

[5] Boucher, R. F., Boysan, F., and Haider, H. S., 1988, "Theoretical Computer Simulations of Swirling Flow in a Vortex Amplifier Chamber," Proceedings of the $2^{\text {nd }}$ Symposium on Fluid Control, Measurement, Mechanics and Flow Visualization, pp. 370-375.

[6] Woolhouse, R. J., Tippetts, J. R., and Beck, S. B. M. A., 2001, “Comparison of the Experimental and Computational Modelling of the Fluidic Turn-up Vortex Amplifier at Full and Zero Swirl Conditions," Proc. Inst. Mech. Eng., Part C: J. Mech. Eng. Sci., 215(8), pp. 893-903.

[7] Aljuwayhel, N. F., Nellis, G. F., and Klein, S.A., 2004, "Parametric and Internal Study of the Vortex Tube Using a CFD Model," Int. J. Refrig., 28, pp. $442-450$.

[8] Upendra-Behera, P. J., Kasthurirengan, S., Karunanithi, R., Ram, S. N., Dinesh, K., and Jacob, S., 2005, "CFD Analysis and Experimental Investigations Towards Optimizing the Parameters of a Ranque-Hilsch Vortex Tube," Int. J.Heat Mass Transfer, 48, pp. 1961-1973.

[9] Wang, J., and Priestman, G. H., 2009, "Flow Simulation in Complex Fluidics Using Three Turbulence Models and Unstructured Grids," Int. J. Numer Methods Heat Fluid Flow, 19(3-4), pp. 484-500. 
[10] ANSYS, 2006, "ANSYS CFX-Solver Theory Guide, ANSYS CFX, Release 11.0." ANSYS Europe Ltd.

[11] MARNET, "Best Practice Guidelines for Marine Applications of Computational Fluid Dynamics," https://pronet.wsatkins.co.uk/marnet/

[12] The American Institute of Aeronautics and Astronautics (AIAA), 1998, "Guide for the Verification and Validation of Computational Fluid Dynamic Simulations," Report No. G-077-1998.

[13] Roache, P. J., 1977, "Quantification of Uncertainty in Computational Fluid Dynamics," Annu. Rev. Fluid Mech., 29, pp. 123-160.

[14] MacGregor, S. A., Syred, N., Markland, E., 1982, "Instabilities Associated With the Outlet Flow in Vortex Amplifiers," Fluidics Q., 14(4), pp. 29-37.

[15] Karagoz, I., and Kaya, F., 2007, "CFD Investigation of the Flow and Heat Transfer Characteristics in a Tangential Inlet Cyclone," Int. Commun. Heat Mass Transfer, 34, pp. 1119-1126.

[16] Roache, P. J., 1994, "A Method for Uniform Reporting of Grid Refinement Studies,” J. Fluid Eng., 116, pp. 405-413.

[17] Syred, N., and Royle, J. K., 1971, "Operating Characteristics of High Performance Vortex Amplifiers," Second IFAC Symposium on Fluidics, Prague, B5, pp. 1-18.
[18] Savino, J. H., and Keshock, E.G., 1965, "Experimental Profile of Velocity Components and Radial Pressure Distributions in a Vortex Contained in a Short Cylindrical Chamber," Proceedings of the 3rd Fluid Amplification Symposium, Harry Diamond Laboratories, Washington, DC

[19] Wormley, D. N., 1976, "A Review of Vortex Diode and Triode Static and Dynamic Design Techniques," AGARD Fluidics Technol., 8, pp. 83-112.

[20] Stairmand, J. W., 1990, "Flow Patterns in Vortex Chambers for Nuclear Duties," J. Nucl. Energy, 29, pp. 413-418.

[21] Peng, W., Hoffmann, A. C., Boot, P. J. A. J., Udding, A., Dries, H. W. A., Ekker, A., and Kater, J., 2002, "Flow Pattern in Reverse-Flow Centrifugal Separators," Powder Technol., 127, pp. 212-222.

[22] King, C. F., 1979, "Some Studies of Vortex Devices-Vortex Amplifier Performance and Behavior," Ph.D. Thesis, University College Cardiff, Cardiff, UK.

[23] Syred, N., 1969, "An Investigation of High Performance Vortex Valves and Amplifiers," Ph.D. Thesis, University of Sheffield, Sheffield, UK.

[24] MacGregor, S. A., and Syred, N., 1982, "Effect of Outlet Diffusers on Vortex Amplifier Characteristics," Fluidics Q., 14, pp. 1-11.

[25] Bendix Aviation Corporation, 1968, UK Patent GB1134489. 